Part of Journal of Research of the National Bureau of Standards, Volume 21, August 1938

\title{
PRELIMINARY LISTS OF TERMS FOR THE ARC AND SPARK SPECTRA OF TUNGSTEN
}

\author{
By Donald D. Laun
}

\section{ABSTRACT}

The new large quartz-prism spectrograph of the National Bureau of Standards has been used to photograph the arc and spark spectra of tungsten in the ultraviolet between 3100 and $2100 \mathrm{~A}$. The wave-length data derived from these observations and also the Zeeman effect observations made with the same instrument, have made it possible to establish the low terms ${ }^{6} \mathrm{D}$ and ${ }^{6 \mathrm{~S}}$ of $\mathrm{W}$ II, together with several other metastable terms whose identity is not yet certain. The raie ultime of $\mathrm{W}_{\mathrm{II}}$ is probably the intense line at $2204.49 \mathrm{~A}$. In $\mathrm{W}_{\mathrm{I}}, 89$ new levels have been found. The $g$-values, derived from new Zeeman effects, are given for 37 levels of $\mathrm{W}$.

\section{CONTENTS}

I. Introduction -207

II. Experimental___.

III. Results_._.

1. First spark spectrum of tungsten W $\mathrm{WI}$

2. New levels and $g$-values for the arc spectrum $\mathrm{W} \mathrm{I}_{\ldots} \ldots$

\section{INTRODUCTION}

Because of their complex character, the spectra of tungsten and uranium, in the sixth column of the periodic system, have been only incompletely described. This circumstance has been largely responsible for the delay in working out the term structure of these spectra. In fact, no terms for uranium spectra are as yet known; but for the first spectrum of tungsten a beginning was made by Laporte ${ }^{1} 13$ years ago, when he announced the low ${ }^{5} \mathrm{D}$ and ${ }^{7} \mathrm{~S}$ terms of $\mathrm{W} \mathrm{I}$, and showed that the two lowest components of ${ }^{7} \mathrm{D}$ actually lie below ${ }^{7} \mathrm{~S}$, contrary to the term arrangement of the analogous spectra Cri and MoI. With the help of new wave-length and Zeeman effect data, the low terms of W II have now been found. It is the purpose of this paper to communicate the new terms, to report the results of some Zeeman effect observations that have been made of ultraviolet lines, and to give the classification of nearly 500 lines that originate in the low terms and also in some metastable terms lying considerably higher than the ground term.

\section{EXPERIMENTAL}

Shortly after Laporte made known his results for the are spectrum, I began a search among the spark lines of tungsten for the ${ }^{6} \mathrm{D}$ and ${ }^{6} \mathrm{~S}$ terms of W II, to correspond with the similar terms which Kiess

\footnotetext{
${ }^{1}$ O. Laporte, Naturwissenschaften 13, 627 (1925).
} 
and Laporte ${ }^{2}$ had established for Crir. But these initial efforts proved fruitless owing to the inadequate descriptions of the ultraviolet portions of the tungsten spectra then available. Accordingly, I undertook to reobserve these spectra, using for this purpose the Hilger E1 quartz spectrograph of the National Bureau of Standards, which could be effectively employed down to 1900 A. Subsequently, these earlier spectrograms have been supplemented with a new series obtained by C. C. Kiess with the large quartz spectrograph, and also with the 20,000 lines per-inch concave grating spectrograph. Both of these instruments have been described elsewhere in this Journal. ${ }^{3}$

In addition to the spectrograms that were obtained for wavelength measurement, Kiess has also made a set of Zeeman effect observations of the ultraviolet tungsten spectra, using the same large quartz spectrograph in conjunction with the water-cooled magnet of the National Bureau of Standards. Equipped with ferro-cobalt polepieces, this magnet supplied a field of 34,500 gausses across a $6-\mathrm{mm}$ gap of circular area $1 \mathrm{~cm}$ in diameter. A current of $150 \mathrm{amp}$ was required to maintain the field.

Small rods of very pure tungsten metal were used as electrodes in the arcs and sparks that served as light sources. Each spectrogram was exposed also to iron or copper arcs to obtain the necessary standards for the reduction of the measurements. The wave lengths of the lines used as standards were taken from the lists of Burns and Walters, ${ }^{4}$ except for two observations of the region 3750 to $3000 \mathrm{~A}$, for which the large grating was used. Here the standards used in the reductions were calculated values of tungsten arc lines observed by Belke. The reason for this procedure was to fit the new wave lengths to the scale of Belke's observations, which Laporte and Mack have used in setting up their system of levels in the analysis of W $\mathrm{I}$.

The spectrograms were all measured and reduced according to the usual procedure. The earlier plates were measured on the large comparator of the Bureau, but the later plates were measured on the comparator of the Physics Department of Marquette University.

\section{RESULTS}

\section{FIRST SPARK SPECTRUM OF TUNGSTEN W II}

In tables 1 and 2 are given the terms which, thus far, have been found for W II. The character of the terms ${ }^{6} \mathrm{D}$ and ${ }^{6} \mathrm{~S}$ is established with certainty by the Zeeman effects. These terms arise, respectively, from the electron configurations $d^{4} s$ and $d^{5}$, for which $L S$-coupling holds, as indicated by the excellent agreement between the observed $g$-values and those calculated from Landé's theory. ${ }^{5}$ For the terms which are clearly identified the usual symbols are employed; the other terms, in accord with a scheme adopted by Laporte and Mack for $\mathrm{W}_{\mathrm{I}}$, are designated by the first two significant figures of their numerical values in the case of the even terms, and by the first three figures in the case of the odd terms, with subscripts denoting the inner quantum numbers, $J$.

\footnotetext{
${ }^{2}$ C. C. Kiess and O. Laporte, Science 63, 234 (1926)

3 C. C. Kiess and E. Z. Stowell, BS J. Research 12, 459 (1934) RP671. W. F. Meggers and K. Burns, BS Sci. Pap. 18, 191 (1923) S 441.

${ }^{4} \mathrm{~K}$. Burns and F. M. Walters, Pub. Allegheny Obs. 8, 27 and 39 (1931).

5 A. Landé, Z. Physik. 15, 189 (1923).
} 
In table 3 are listed 500 lines that have been classified on the basis of the terms in tables 1 and 2. Following each wave length are, in order, the estimated intensities of the lines in the arc and spark sources, the wave number, the term combination, and, lastly, the observed Zeeman effect. For all the lines, except the first four, the estimated intensities are from National Bureau of Standards spectrograms. For the first four lines the intensities $B$ are from Belke ${ }^{6}$ and the intensities $E$ from Exner and Hascheck. The letters following the intensities describe the following characteristics of the lines: $a$, classified also in W I; $b$, broadened; $c$, complex; $d$, double; $r$, reversed; $p$, apparent pole-effect or transverse variation of intensity ( $p r$ reversed only at end of line); $l$, displaced toward the red; and $v$, displaced toward the violet.

TABLE 1.-Even terms of W II

\begin{tabular}{|c|c|c|c|c|}
\hline \multirow{2}{*}{\multicolumn{2}{|c|}{$\begin{array}{c}\text { Term } \\
\text { symbol }\end{array}$}} & \multirow{2}{*}{ Term value } & \multicolumn{2}{|c|}{$g$-value } \\
\hline & & & observed & theoretical \\
\hline & $\begin{array}{l}{ }^{6} \mathrm{D}_{01 / 2} \\
{ }^{6} \mathrm{D}_{11 / 3} \\
{ }^{6} \mathrm{D}_{21 / 2} \\
{ }^{6} \mathrm{D}_{31 / 2} \\
{ }^{6} \mathrm{D}_{41 / 2}\end{array}$ & $\begin{array}{r}0.0 \\
\text { 1518. } 8 \\
3172.7 \\
4716.4 \\
6147.1\end{array}$ & $\begin{array}{l}\text { 3. } 20 \\
\text { 1. } 86 \\
\text { 1. } 64 \\
\text { 1. } 58 \\
\text { 1. } 56\end{array}$ & $\begin{array}{l}\text { 3. } 33 \\
1.87 \\
1.66 \\
1.59 \\
1.56\end{array}$ \\
\hline$d^{5}$ & $\begin{array}{l}{ }^{6} \mathrm{~S}_{21 / 2} \\
{ }^{4} \mathrm{~F}_{11 / 3} \\
08_{01 / 3} \\
13_{01 / 3} \\
10_{11 / 2}\end{array}$ & $\begin{array}{r}7420.5 \\
8711.3 \\
8832.7 \\
13173.3 \\
10592.5\end{array}$ & $\begin{array}{l}\text { 1. } 94 \\
0.64 \\
2.5 \\
0.48\end{array}$ & $\begin{array}{l}\text { 2. } 00 \\
0.40\end{array}$ \\
\hline & $\begin{array}{l}14_{11 / 2} \\
11_{21 / 2} \\
13_{21 / 2} \\
14_{21 / 3} \\
16_{21 / 2}\end{array}$ & $\begin{array}{l}\text { 14634. } 4 \\
11301.0 \\
13434.2 \\
14967.9 \\
16234.9\end{array}$ & $\begin{array}{l}\text { 1. } 2 \\
\text { 1. } 1 \\
\text { 1. } 4\end{array}$ & \\
\hline & $\begin{array}{l}23_{21 / 3} \\
13_{31 / 3} \\
15_{31 / 3} \\
16_{31 / 2} \\
20_{31 / 2}\end{array}$ & $\begin{array}{l}23450.4 \\
13412.0 \\
15147.0 \\
16589.7 \\
20039.8\end{array}$ & $\begin{array}{l}\text { 1. } 2 \\
\text { 1. } 0 \\
\text { 1. } 3\end{array}$ & \\
\hline & $\begin{array}{l}14_{41 / 2} \\
16_{41 / 2} \\
19_{41 / 2} \\
20_{41 / 2} \\
23_{41 / 2}\end{array}$ & $\begin{array}{l}14857.2 \\
16553.1 \\
19070.9 \\
20780.5 \\
23234.8\end{array}$ & $\begin{array}{l}\text { 1. } 2 \\
\text { 1. } 1\end{array}$ & \\
\hline & $\begin{array}{l}17_{51 / 2} \\
20_{51 / 2}\end{array}$ & $\begin{array}{l}\text { 17437. } 1 \\
20534.4\end{array}$ & 1. 2 & \\
\hline
\end{tabular}

${ }^{6}$ M. Belke, Z. wiss. Phot. 17, 132 and 145 (1918). 
TABLE 2.-Odd terms of W II

\begin{tabular}{|c|c|c|c|c|c|}
\hline $\begin{array}{c}\text { Term } \\
\text { symbol }\end{array}$ & $\begin{array}{l}\text { Term } \\
\text { value }\end{array}$ & $\begin{array}{c}\text { Ob- } \\
\text { served } \\
g \text {-value }\end{array}$ & $\begin{array}{c}\text { Term } \\
\text { symbol }\end{array}$ & $\begin{array}{l}\text { Term } \\
\text { value }\end{array}$ & $\underset{\text { Served }}{\text { Ob-value }}$ \\
\hline $\begin{array}{l}361_{01 / 2} \\
385_{01 / 3} \\
444_{01 / 2} \\
454_{01 / 2} \\
523_{01 / 2}\end{array}$ & $\begin{array}{l}36165.3 \\
38576.3 \\
44455.2 \\
45457.0 \\
52355.0\end{array}$ & $\begin{array}{c}0.67 \\
1.67 \\
.2 \\
.4\end{array}$ & $\begin{array}{l}448_{31 / 2} \\
461_{31 / 2} \\
488_{31 / 2} \\
491_{31 / 2} \\
518_{31 / 2}\end{array}$ & $\begin{array}{l}44877.1 \\
46175.4 \\
48830.7 \\
49124.5 \\
51863.0\end{array}$ & $\begin{array}{l}\text { 1. } 32 \\
1.0 \\
.9\end{array}$ \\
\hline $\begin{array}{l}391_{11 / 2} \\
422_{1 / 2} \\
449_{1 / 1} \\
471_{1 / 2} \\
533_{11 / 2}\end{array}$ & $\begin{array}{l}39129.4 \\
42298.1 \\
44911.7 \\
47180.0 \\
53329.7\end{array}$ & $\begin{array}{l}1.14 \\
1.50 \\
1.24 \\
1.04\end{array}$ & $\begin{array}{l}522_{31 / 2} \\
529_{31 / 2} \\
533_{31 / 2} \\
577_{31 / 2} \\
592_{31 / 2}\end{array}$ & $\begin{array}{l}52275.5 \\
52901.7 \\
53338.0 \\
57730.0 \\
59276.8\end{array}$ & \\
\hline $\begin{array}{l}534_{11 / 2} \\
541_{11 / 2} \\
560_{11 / 2} \\
420_{21 / 2} \\
443_{21 / 2}\end{array}$ & $\begin{array}{l}53423.0 \\
54137.3 \\
56084.3 \\
42049.4 \\
44354.8\end{array}$ & $\begin{array}{l}1.6 \\
\text { 1. } 30 \\
1.40\end{array}$ & $\begin{array}{l}598_{31 / 2} \\
447_{41 / 2} \\
464_{41 / 2} \\
491_{41 / 2} \\
508_{41 / 3}\end{array}$ & $\begin{array}{l}59869.1 \\
44758.0 \\
46493.4 \\
49181.0 \\
50863.1\end{array}$ & $\begin{array}{l}\text { 1. } 30 \\
\text { 1. } 32 \\
\text { 1. } 2\end{array}$ \\
\hline $\begin{array}{l}463_{21 / 2} \\
474_{21 / 2} \\
482_{21 / 2} \\
492_{21 / 2} \\
514_{21 / 2}\end{array}$ & $\begin{array}{l}46355.4 \\
47413.3 \\
48284.6 \\
49242.2 \\
51438.0\end{array}$ & $\begin{array}{l}\text { 1. } 2 \\
1.14 \\
1.38 \\
1.5 \\
1.3\end{array}$ & $\begin{array}{l}533_{41 / 2} \\
540_{41 / 2} \\
553_{41 / 2} \\
593_{41 / 2} \\
514_{51 / 2}\end{array}$ & $\begin{array}{l}53370.0 \\
54056.6 \\
55392.5 \\
59399.4 \\
51495.0\end{array}$ & 1. 2 \\
\hline $\begin{array}{l}531_{21 / 2} \\
547_{21 / 2} \\
567_{21 / 2} \\
568_{21 / 2} \\
423_{31 / 2}\end{array}$ & $\begin{array}{l}53113.6 \\
54704.7 \\
56768.7 \\
56875.1 \\
42390.2\end{array}$ & 1. 16 & $\begin{array}{l}549_{51 / 2} \\
588_{51 / 2} \\
602_{51 / 2} \\
612_{51 / 2} \\
615_{51 / 2}\end{array}$ & $\begin{array}{l}54958.6 \\
58891.8 \\
60218.9 \\
61240.8 \\
61589.6\end{array}$ & $\begin{array}{l}1.1 \\
1.2 \\
1.1\end{array}$ \\
\hline
\end{tabular}

TABLE 3.-Classified lines of W II

\begin{tabular}{|c|c|c|c|c|c|}
\hline \multirow{2}{*}{$\lambda_{\mathrm{air}}$} & \multicolumn{2}{|c|}{ Intensity } & \multirow{2}{*}{$\begin{array}{c}\text { Wave } \\
\text { number }\end{array}$} & \multirow{2}{*}{$\begin{array}{c}\text { Term } \\
\text { combination }\end{array}$} & \multirow{2}{*}{ Zeeman effect } \\
\hline & Arc & Spark & & & \\
\hline $\begin{array}{l}4348.13 \\
4175.63 \\
3935.44 \\
3851.57 \\
3716.08\end{array}$ & $\begin{array}{l}\mathrm{B} 2 \\
\mathrm{E} 6 \\
\mathrm{~B} 1 \\
\mathrm{~B} 1 \\
10\end{array}$ & $\begin{array}{l}\text { E8 } \\
\text { E8 } \\
\text { E10 } \\
\text { E6 } \\
50\end{array}$ & $\begin{array}{l}22992.0 \\
23941.8 \\
25403.0 \\
25956.1 \\
26902.4\end{array}$ & $\begin{array}{l}13_{01 / 2}-361_{01 / 2} \\
14_{11 / 2}-385_{01 / 2} \\
13_{01 / 2}-385_{01 / 2} \\
13_{01 / 2}-391_{11 / 2} \\
15_{31 / 2}-420_{21 / 2}\end{array}$ & $\begin{array}{ll}(0.00) & 0.57 \mathrm{~J} \\
(0.24) & 1.49,1.96 \mathrm{Be} \\
(0.59) & 1.07 \mathrm{~J} \\
(0.00) & 1.53 \mathrm{~J}\end{array}$ \\
\hline $\begin{array}{l}3691.49 \\
3688.34 \\
3657.88 \\
3657.59 \\
3652.14\end{array}$ & $\begin{array}{l}10 l \\
10 \\
40 \\
12 b\end{array}$ & $\begin{array}{c}4 \\
4 \\
30 \\
120 \\
10 b\end{array}$ & $\begin{array}{l}27081.6 \\
27104.8 \\
27330.5 \\
27332.6 \\
27373.4\end{array}$ & $\begin{array}{l}14_{21 / 3}-420_{21 / 3} \\
19_{41 / 1}-461_{31 / 2} \\
14_{21 / 3}-422_{11 / 2} \\
08_{01 / 2}-361_{01 / 2} \\
20_{31 / 2}-474_{21 / 3}\end{array}$ & $(0.88) 1.56 \mathrm{~J}$ \\
\hline $\begin{array}{l}3646.60 \\
3645.61\end{array}$ & $\begin{array}{l}10 \\
10\end{array}$ & $\begin{array}{l}40 \\
40\end{array}$ & $\begin{array}{l}27415.0 \\
27422.5\end{array}$ & $\left\{\begin{array}{l}14_{11 / 2}-420_{21 / 2} \\
19_{41 / 2}-464_{41 / 2} \\
14^{2}-423^{21}\end{array}\right.$ & $(0.00) 1.37 \mathrm{~J}$ \\
\hline $\begin{array}{l}3641.42 \\
3630.98 \\
3618.46\end{array}$ & $\begin{array}{r}40 \\
3\end{array}$ & $\begin{array}{r}150 \\
5 \\
30\end{array}$ & $\begin{array}{l}27454.0 \\
27533.0 \\
27628.2\end{array}$ & $\begin{array}{l}{ }^{4} \mathrm{~F}_{11 / 2}-361_{01 / 2} \\
14_{41 / 2}-423_{31 / 2} \\
23_{41 / 2}-508_{41 / 2}\end{array}$ & $(0.00) 0.60 \mathrm{~J}$ \\
\hline
\end{tabular}


TABLE 3.-Classified lines of $\mathrm{W} \mathrm{II}-$ Continued

\begin{tabular}{|c|c|c|c|c|c|}
\hline \multirow{2}{*}{$\lambda_{\text {air }}$} & \multicolumn{2}{|c|}{ Intensity } & \multirow{2}{*}{$\begin{array}{l}\text { Wave } \\
\text { number }\end{array}$} & \multirow{2}{*}{$\begin{array}{c}\text { Term } \\
\text { combination }\end{array}$} & \multirow{2}{*}{ Zeeman effect } \\
\hline & Arc & Spark & & & \\
\hline $\begin{array}{l}3613.80 \\
3592.43 \\
3572.48 \\
3572.03 \\
3563.98\end{array}$ & $\begin{array}{c}30 \\
50 \\
100 \\
8 v\end{array}$ & $\begin{array}{r}80 \\
80 \\
200 \\
10 \\
3\end{array}$ & $\begin{array}{l}27663.9 \\
27828.4 \\
27983.8 \\
27987.5 \\
28050.5\end{array}$ & $\begin{array}{l}14_{11 / 2}-422_{11 / 2} \\
11_{21 / 2}-391_{11 / 1} \\
10_{11 / 2}-385_{01 / 2} \\
23_{21 / 2}-514_{21 / 2} \\
20_{43 / 2}-488_{31 / 2}\end{array}$ & $\begin{array}{l}(0.40) 1.40 \mathrm{~J} ; 3 / 2-3 / 2 \mathrm{Be} \\
(0.00) 1.13 \mathrm{~J}\end{array}$ \\
\hline $\begin{array}{l}3555.18 \\
3549.08 \\
3544.48 \\
3539.48 \\
3534.14\end{array}$ & $\begin{array}{l}12 \\
30 d ? \\
3 \\
3\end{array}$ & $\begin{array}{r}120 \\
150 \\
30 \\
15 \\
10\end{array}$ & $\begin{array}{l}28120.0 \\
28168.3 \\
28204.8 \\
28244.7 \\
28287.4\end{array}$ & $\begin{array}{l}16_{21 / 2}-443_{21 / 3} \\
16_{31 / 2}-4477_{41 / 2} \\
16_{41 / 2}-4477_{41 / 5} \\
20_{31 / 2}-482_{21 / 3} \\
16_{31 / 2}-448_{31 / 2}\end{array}$ & $\begin{array}{l}(0.93) \\
(0.00) \\
(.51 \mathrm{~J}\end{array}$ \\
\hline $\begin{array}{l}3529.57 \\
3527.05 \\
3518.57 \\
3503.24 \\
3492.08\end{array}$ & $\begin{array}{l}60 r ? ? \\
1 \\
1\end{array}$ & $\begin{array}{r}100 \\
40 \\
3 \\
4 \\
40\end{array}$ & $\begin{array}{l}28324.0 \\
28344.2 \\
28412.5 \\
28536.9 \\
28628.1\end{array}$ & $\begin{array}{l}16_{41 / 2}-448_{31 / 2} \\
20_{41 / 2}-491_{31 / 2} \\
23_{21 / 3}-518_{31 / 2} \\
10_{11 / 2}-391_{11 / 2} \\
23_{41 / 3}-518_{31 / 2}\end{array}$ & $\begin{array}{l}(0.00) \\
(0.00) \\
0.97 \mathrm{~J}\end{array}$ \\
\hline $\begin{array}{l}3490.92 \\
3490.33 \\
3489.81 \\
3486.14 \\
3472.33\end{array}$ & $\begin{array}{r}12 \\
8 \\
1 \\
10 \\
1\end{array}$ & $\begin{array}{r}80 \\
50 \\
10 \\
100 \\
15\end{array}$ & $\begin{array}{l}28637.6 \\
28642.4 \\
28646.7 \\
28676.8 \\
28790.9\end{array}$ & $\begin{array}{l}13_{31 / 2}-420_{21 / 2} \\
16_{21 / 2}-448_{31 / 2} \\
20_{51 / 2}-491_{41 / 2} \\
16_{21 / 2}-449_{11 / 2} \\
20_{31 / 2}-488_{31 / 2}\end{array}$ & $(0.00) 1.00 \mathrm{~J}$ \\
\hline $\begin{array}{l}3468.24 \\
3463.52 \\
3452.51 \\
3449.88 \\
3442.52\end{array}$ & $\begin{array}{c}6 v \\
20 v \\
6 \\
8 \\
2\end{array}$ & $\begin{array}{c}10 \\
200 \\
30 \\
80 \\
20 ?\end{array}$ & $\begin{array}{l}28824.8 \\
28864.1 \\
28956.2 \\
28978.2 \\
29040.2\end{array}$ & $\begin{array}{l}23_{21 / 3}-522_{31 / 2} \\
13_{21 / 2}-422_{11 / 2} \\
13_{21 / 2}-423_{31 / 3} \\
13_{31 / 2}-423_{31 / 3} \\
23_{41 / 2}-522_{31 / 2}\end{array}$ & $\begin{array}{l}(0.00) 1.47 \mathrm{~J} \\
(0.00) 1.28 \mathrm{~J}\end{array}$ \\
\hline $\begin{array}{l}3440.60 \\
3437.25 \\
3430.57 \\
3401.90 \\
3394.47\end{array}$ & $\begin{array}{r}30 \\
1\end{array}$ & $\begin{array}{c}20 \\
20 b ? \\
2 \\
150 \\
20\end{array}$ & $\begin{array}{l}\text { 29056. } 4 \\
29084.7 \\
\text { 29141. } 4 \\
29386.9 \\
29451.3\end{array}$ & $\begin{array}{l}17_{51 / 2}-464_{41 / 2} \\
20_{31 / 2}-491_{31 / 2} \\
20_{31 / 2}-491_{41 / 2} \\
14_{21 / 2}-443_{21 / 2} \\
23_{21 / 2}-529_{31 / 2}\end{array}$ & $\begin{array}{l}(0.00) 1.00 \mathrm{~J} \\
(0.79) 1.25 \mathrm{~J}\end{array}$ \\
\hline $\begin{array}{l}3379.04 \\
3376.17\end{array}$ & $\begin{array}{r}2 \\
20\end{array}$ & $\begin{array}{r}50 \\
400\end{array}$ & $\begin{array}{l}29585.7 \\
29610.9\end{array}$ & $\begin{array}{l}16_{31 / 2}-461_{31 / 2} \\
15_{31 / 2}-447_{41 / 2}\end{array}$ & $\underset{9 / 2}{(0.00)} \underset{\mathrm{Be}}{2.14} \mathrm{~J} ; 7 / 2-$ \\
\hline $\begin{array}{l}3374.87 \\
3370.25 \\
3369.81\end{array}$ & $1 b$ & $\begin{array}{l}30 \\
5 \\
6 b l\end{array}$ & $\begin{array}{l}29622.3 \\
29662.9 \\
29666.8\end{array}$ & $\begin{array}{l}16_{41 / 2}-461_{31 / 2} \\
23_{21 / 2}-531_{21 / 2} \\
23_{41 / 2}-529_{31 / 2}\end{array}$ & \\
\hline $\begin{array}{l}3363.73 \\
3362.60\end{array}$ & $15 c$ & 40 & 29720.4 & $\begin{array}{l}14_{11 / 1}-443_{21 / 2} \\
15_{31 / 2}-448_{31 / 2}\end{array}$ & \\
\hline 3361. 11 & 40 & 100 & 29743. 6 & $08_{01 / 2}-385_{01 / 2}$ & $\begin{array}{c}(0.00) 2.01 \mathrm{~J} ;(0.82) \\
2.50 \mathrm{Be}\end{array}$ \\
\hline $\begin{array}{l}3359.28 \\
3358.62\end{array}$ & 70 & $\begin{array}{r}30 \\
200\end{array}$ & $\begin{array}{l}29759.8 \\
29765.6\end{array}$ & $\begin{array}{l}19_{41 / 2}-488_{31 / 2} \\
16_{31 / 2}-463_{21 / 2}\end{array}$ & $(0.00) 1.11 \mathrm{~J}$ \\
\hline $\begin{array}{l}3352.38 \\
3347.47 \\
3345.86 \\
3344.89 \\
3343.40\end{array}$ & $\begin{array}{r}3 c \\
200 \\
5 \\
12\end{array}$ & $\begin{array}{c}30 b \\
3 b \\
200 b \\
25 \\
120\end{array}$ & $\begin{array}{l}29821.0 \\
29864.8 \\
29879.1 \\
29887.8 \\
29901.1\end{array}$ & $\begin{array}{l}14_{11 / 1}-444_{01 / 2} \\
{ }^{4} \mathrm{~F}_{11 / 2}-385_{01 / 2} \\
23_{21 / 1}-533_{11 / 2} \\
23_{21 / 2}-533_{31 / 2} \\
14_{41 / 2}-447_{41 / 2}\end{array}$ & $\begin{array}{l}(0.00) .93 \mathrm{~J} \\
(0.00) 1.12 \mathrm{~J}\end{array}$ \\
\hline
\end{tabular}


Table 3.-Classified lines of W II-Continued

\begin{tabular}{|c|c|c|c|c|c|}
\hline \multirow{2}{*}{$\lambda_{\mathrm{air}}$} & \multicolumn{2}{|c|}{ Intensity } & \multirow{2}{*}{$\begin{array}{l}\text { Wave } \\
\text { number }\end{array}$} & \multirow{2}{*}{$\begin{array}{c}\text { Term } \\
\text { combination }\end{array}$} & \multirow{2}{*}{ Zeeman effect } \\
\hline & Arc & Spark & & & \\
\hline $\begin{array}{l}\text { 3343. } 09 \\
3342.46\end{array}$ & $\begin{array}{l}10 \\
30\end{array}$ & $\begin{array}{l}100 \\
300\end{array}$ & $\begin{array}{l}29903.9 \\
29909.5\end{array}$ & $\begin{array}{l}16_{31 / 2}-464_{41 / 2} \\
14_{21 / 2}-448_{31 / 2}\end{array}$ & $\begin{array}{ll}(0.00) & 1.41 \mathrm{~J} \\
(0.00) & 1.70 \mathrm{~J}\end{array}$ \\
\hline 3339. 03 & 5 & 60 & 29940.2 & $\left\{\begin{array}{l}16_{41 / 2}-464_{41 / 2} \\
16\end{array}\right.$ & \\
\hline $\begin{array}{l}3338.63 \\
3335.40\end{array}$ & 5 & $\begin{array}{c}50 \\
5 b\end{array}$ & $\begin{array}{l}29943.8 \\
29972.8\end{array}$ & $\begin{array}{l}14_{21 / 2}-449_{11 / 2} \\
23_{21 / 2}-534_{11 / 2}\end{array}$ & \\
\hline $\begin{array}{l}\text { 3320. } 94 \\
3317.41 \\
3301.85 \\
3299.74 \\
3286.57\end{array}$ & $\begin{array}{c}2 \\
18 \mathrm{cv} \\
6 \\
20\end{array}$ & $\begin{array}{r}20 b \\
25 b \\
20 \\
30 \\
100\end{array}$ & $\begin{array}{l}\text { 30103. } 3 \\
30135.4 \\
30277.4 \\
30296.7 \\
\text { 30418. } 1\end{array}$ & $\begin{array}{l}23_{41 / 2}-533_{31 / 2} \\
23_{31 / 2}-533_{41 / 2} \\
14_{1 / 2}-449_{11 / 2} \\
08_{012}-391_{11 / 2} \\
{ }^{4} \mathrm{~F}_{11 / 2}-391_{11 / 2}\end{array}$ & \\
\hline $\begin{array}{l}3257.81 \\
3254.85 \\
3251.25\end{array}$ & $\begin{array}{l}? \\
1 c \\
?\end{array}$ & $\begin{array}{l}20 b \\
10 b \\
50 c l\end{array}$ & $\begin{array}{l}30686.6 \\
30714.6 \\
30748.6\end{array}$ & $\begin{array}{l}23_{21 / 2}-541_{11 / 2} \\
20_{41 / 2}-514_{51 / 2} \\
11_{21 / 2}-420_{21 / 2}\end{array}$ & \\
\hline 3243. 48 & 1 & $4 b$ & 30822. 2 & & \\
\hline 3243.36 & $20 v$ & 100 & 30823. 4 & $\left\{\begin{array}{l}16_{31 / 2}-474_{21 / 2} \\
20_{31 / 2}-508_{41 / 2}\end{array}\right.$ & $(0.00) 1.24 \mathrm{~J}$ \\
\hline $\begin{array}{l}3233.15 \\
3230.85 \\
3230.61 \\
3228.99 \\
3225.18\end{array}$ & $\begin{array}{c}3 \\
15 l \\
5 p \\
8 c l \\
1\end{array}$ & $\begin{array}{l}15 b \\
15 \\
25 \\
20 b \\
4 b\end{array}$ & $\begin{array}{l}\text { 30920. } 7 \\
30942.7 \\
30945.0 \\
30960.5 \\
30997.1\end{array}$ & $\begin{array}{l}13_{21 / 2}-443_{21 / 2} \\
13_{31 / 2}-443_{21 / 2} \\
16_{21 / 2}-471_{11 / 2} \\
20_{51 / 2}-514_{51 / 2} \\
11_{21 / 2}-422_{11 / 2}\end{array}$ & \\
\hline $\begin{array}{l}\text { 3216. } 32 \\
\text { 3215. } 67 \\
\text { 3206. } 42 \\
\text { 3203. } 44 \\
3203.34\end{array}$ & $\begin{array}{l}2 \\
? \\
8 ? \\
8 v \\
12\end{array}$ & $\begin{array}{l}30 \\
30 \\
80 \\
40 \\
80\end{array}$ & $\begin{array}{l}31082.5 \\
31088.8 \\
31178.4 \\
31207.5 \\
31208.4\end{array}$ & $\begin{array}{l}20_{41 / 2}-518_{31 / 2} \\
11_{21 / 2}-423_{31 / 2} \\
16_{21 / 2}-474_{21 / 2} \\
14_{21 / 2}-461_{31 / 2} \\
15_{31 / 2}-463_{21 / 2}\end{array}$ & \\
\hline 3192. 11 & & $15 b c$ & 31318. 2 & $14_{41 / 2}-461_{31 / 5}$ & \\
\hline 3189. 24 & $50 a$ & $100 l$ & 31346. 4 & $\left\{\begin{array}{l}13_{31 / 2}-447_{41 / 1} \\
15_{315}-464_{414}\end{array}\right.$ & \\
\hline $\begin{array}{l}3185.06 \\
3183.97 \\
3179.44\end{array}$ & $\begin{array}{c}3 d \\
4 \\
15\end{array}$ & $\begin{array}{r}30 \\
10 \\
150\end{array}$ & $\begin{array}{l}31387.5 \\
31398.3 \\
31443.0\end{array}$ & $\begin{array}{l}14_{21 / 2}-463_{21 / 2} \\
20_{31 / 2}-514_{21 / 2} \\
13_{21 / 2}-448_{31 / 2}\end{array}$ & \\
\hline $\begin{array}{l}3178.04 \\
3177.22 \\
3175.97 \\
3160.03 \\
3154.19\end{array}$ & $\begin{array}{l}8 ? \\
60 d(r) \\
20 \\
20 \\
6\end{array}$ & $\begin{array}{r}80 \\
150 \\
200 \\
300 \\
15\end{array}$ & $\begin{array}{l}\text { 31456. } 9 \\
31465.0 \\
31477.4 \\
31636.1 \\
31694.7\end{array}$ & $\begin{array}{l}10_{11 / 2}-420_{21 / 2} \\
13_{31 / 2}-448_{31 / 2} \\
13_{21 / 2}-449_{11 / 2} \\
14_{41 / 2}-464_{41 / 2} \\
16_{31 / 2}-482_{21 / 2}\end{array}$ & $(0.00) 1.38 \mathrm{~J}$ \\
\hline $\begin{array}{l}3153.14 \\
3152.76 \\
3151.58 \\
3151.31 \\
3149.87\end{array}$ & $\begin{array}{r}? \stackrel{?}{6 b} \\
6 \\
6 \\
20\end{array}$ & $\begin{array}{c}3 b \\
10 b \\
20 \\
300 \\
500\end{array}$ & $\begin{array}{l}31705.3 \\
31709.1 \\
31721.0 \\
31723.7 \\
31738.2\end{array}$ & $\begin{array}{l}10_{11 / 2}-422_{11 / 2} \\
6 \mathrm{~S}_{21 / 2}-391_{11 / 2} \\
14_{11 / 2}-463_{21 / 2} \\
23_{41 / 2}-549_{51 / 2} \\
13_{01 / 2}-449_{11 / 2}\end{array}$ & $(0.00) 1.61 \mathrm{~J}$ \\
\hline $\begin{array}{l}3144.50 \\
3108.79 \\
3103.53 \\
3101.23 \\
3100.75\end{array}$ & $\begin{array}{c}10 \\
2 v \\
6 v\end{array}$ & $\begin{array}{l}30 b \\
80 \\
20 \\
6 \\
60\end{array}$ & $\begin{array}{l}31792.4 \\
32157.6 \\
32212.1 \\
32235.9 \\
32240.9\end{array}$ & $\begin{array}{l}19_{41 / 1}-508_{41 / 2} \\
23_{41 / 2}-553_{41 / 2} \\
14_{21 / 2}-471_{11 / 2} \\
20_{31 / 2}-522_{31 / 2} \\
16_{31 / 2}-488_{31 / 3}\end{array}$ & \\
\hline
\end{tabular}


TABle 3.-Classified lines of W II-Continued

\begin{tabular}{|c|c|c|c|c|c|}
\hline \multirow{2}{*}{$\lambda_{\mathrm{air}}$} & \multicolumn{2}{|c|}{ Intensity } & \multirow{2}{*}{$\begin{array}{l}\text { Wave } \\
\text { number }\end{array}$} & \multirow{2}{*}{$\begin{array}{c}\text { Term } \\
\text { combination }\end{array}$} & \multirow{2}{*}{ Zeeman effect } \\
\hline & Arc & Spark & & & \\
\hline $\begin{array}{l}\text { 3098. } 31 \\
\text { 3083. } 29 \\
3081.23 \\
3072.75 \\
3071.74\end{array}$ & $\begin{array}{c}3 \\
1 \\
6 \\
10\end{array}$ & $\begin{array}{c}15 \\
2 \\
4 b \\
40 \\
60\end{array}$ & $\begin{array}{l}32266.3 \\
32423.5 \\
32445.2 \\
32534.7 \\
32545.4\end{array}$ & $\begin{array}{l}15_{31 / 2}-474_{21 / 2} \\
19_{41 / 2}-514_{51 / 2} \\
14_{21 / 2}-474_{21 / 2} \\
16_{31 / 2}-491_{131 / 2} \\
14_{11 / 2}-471_{11 / 2}\end{array}$ & \\
\hline $\begin{array}{l}\text { 3069. } 29 \\
3067.58 \\
3067.42 \\
3066.99 \\
3063.98\end{array}$ & $\begin{array}{l}10 \\
8 \\
4 \\
8(v ?) \\
5\end{array}$ & $\begin{array}{l}90 \\
80 \\
10 \\
80 \\
50\end{array}$ & $\begin{array}{l}32571.4 \\
32589.6 \\
32591.3 \\
32595.8 \\
32627.8\end{array}$ & $\begin{array}{l}16_{41 / 2}-491_{31 / 2} \\
20_{41 / 2}-533_{41 / 2} \\
16_{31 / 2}-491_{41 / 2} \\
16_{21 / 2}-488_{31 / 2} \\
16_{41 / 2}-491_{41 / 2}\end{array}$ & \\
\hline $\begin{array}{l}3063.42 \\
3061.69 \\
3053.36 \\
3051.30 \\
3049.86\end{array}$ & $\begin{array}{l}? \\
10 \\
10 b \\
40 \\
8\end{array}$ & $\begin{array}{c}20 b \\
30 \\
? \\
400 \\
60\end{array}$ & $\begin{array}{l}\text { 32633. } 8 \\
32652.2 \\
32741.3 \\
32763.4 \\
32778.9\end{array}$ & $\begin{array}{l}23_{21 / 2}-560_{11 / 2} \\
16_{31 / 2}-492_{21 / 2} \\
13_{21 / 2}-461_{31 / 2} \\
13_{31 / 2}-461_{31 / 2} \\
14_{11 / 2}-474_{21 / 2}\end{array}$ & \\
\hline $\begin{array}{l}\text { 3048. } 61 \\
3044.57 \\
3039.59 \\
3036.68 \\
3028.74\end{array}$ & $\begin{array}{l}4 \\
3 v \\
8 v\end{array}$ & $\begin{array}{c}40 \\
1 b \\
30 \\
100 \\
15 b\end{array}$ & $\begin{array}{l}\text { 32792. } 3 \\
32835.8 \\
32889.6 \\
32921.2 \\
33007.4\end{array}$ & $\begin{array}{l}19_{41 / 2}-518_{31 / 2} \\
20_{51 / 2}-533_{41 / 3} \\
16_{21 / 2}-491_{33 / 5} \\
13_{21 / 2}-463_{21 / 2} \\
16_{21 / 2}-492_{21 / 2}\end{array}$ & \\
\hline $\begin{array}{l}\text { 3024. } 51 \\
3022.68 \\
3021.98 \\
3010.76 \\
3004.29\end{array}$ & $\begin{array}{l}30 \\
5 b v \\
50 \\
5 \\
7 v\end{array}$ & $\begin{array}{r}300 \\
50 \\
100 \\
100 \\
5\end{array}$ & $\begin{array}{l}\text { 33053. } 6 \\
33073.6 \\
33081.3 \\
33204.5 \\
33276.1\end{array}$ & $\begin{array}{l}11_{21 / 2}-443_{21 / 2} \\
20_{31 / 2}-531_{21 / 2} \\
13_{31 / 2}-464_{41 / 2} \\
19_{41 / 2}-522_{31 / 2} \\
20_{41 / 2}-540_{41 / 2}\end{array}$ & \\
\hline $\begin{array}{l}3002.29 \\
3000.63 \\
3000.50 \\
2998.69 \\
2990.85\end{array}$ & $\begin{array}{r}2 \\
5 \\
-15 \\
- \\
-\end{array}$ & $\begin{array}{c}40 \\
50 \\
3 b \\
50 \\
12 b\end{array}$ & $\begin{array}{l}\text { 33298. } 2 \\
33316.7 \\
33318.1 \\
33338.2 \\
33425.6\end{array}$ & $\begin{array}{l}20_{31 / 2}-533_{31 / 2} \\
14_{21 / 2}-482_{21 / 3} \\
23_{21 / 2}-567_{21 / 3} \\
{ }^{4} \mathrm{~F}_{11 / 2}-420_{21 / 3} \\
17_{51 / 2}-508_{41 / 2}\end{array}$ & $(0.00) 1.98 \mathrm{~J}$ \\
\hline $\begin{array}{l}\text { 2987. } 29 \\
2982.22 \\
2977.44 \\
2976.48 \\
2974.38\end{array}$ & $\begin{array}{r}15 \\
2 \\
-15 \\
20\end{array}$ & $\begin{array}{l}60 \\
25 \\
3 b \\
50 \\
35\end{array}$ & $\begin{array}{l}33465.4 \\
33522.3 \\
33576.1 \\
33587.0 \\
33610.7\end{array}$ & $\begin{array}{l}08_{013}-422_{11 / 1} \\
20_{51 / 2}-540_{41 / 13} \\
11_{21 / 2}-448_{31 / 3} \\
{ }^{4} \mathrm{~F}_{113}-422_{11 / 3} \\
11_{21 / 2}-449_{11 / 2}\end{array}$ & $\begin{array}{l}(0.46) 0.00 b,[1.09,2.04 \\
(1.28) 0.00,1.05,1.95 \\
(0.00) 0.94\end{array}$ \\
\hline $\begin{array}{l}\text { 2970. } 91 \\
\text { 2967. } 92 \\
\text { 2962. } 51 \\
\text { 2961. } 02 \\
\text { 2955. } 01\end{array}$ & $\begin{array}{r}2 \\
4 \\
-12 \\
8\end{array}$ & $\begin{array}{r}5 \\
20 \\
8 b \\
50 \\
15\end{array}$ & $\begin{array}{l}\text { 33649. } 9 \\
33683.8 \\
33745.3 \\
33762.3 \\
33831.0\end{array}$ & $\begin{array}{l}14_{11 / 2}-482_{21 / 2} \\
15_{31 / 2}-488_{31 / 2} \\
13_{21 / 2}-471_{11 / 2} \\
10_{11 / 2}-443_{21 / 2} \\
19_{41 / 2}-529_{31 / 2}\end{array}$ & \\
\hline $\begin{array}{l}\text { 2952. } 26 \\
2942.62 \\
2942.26 \\
2942.13 \\
2940.21\end{array}$ & $\begin{array}{c}75 d ? \\
3 \\
2 \\
12 \\
8\end{array}$ & $\begin{array}{l}100 d ? \\
10 \\
10 \\
10 d \\
60\end{array}$ & $\begin{array}{l}33862.5 \\
\text { 33973. } 4 \\
33977.6 \\
33979.1 \\
34001.3\end{array}$ & $\begin{array}{r}\left\{\begin{array}{r}14_{21 / 3}-488_{31 / 2} \\
10_{11 / 2}-444_{01 / 2}\end{array}\right. \\
14_{41 / 2}-488_{31 / 2} \\
15_{31 / 2}-491_{31 / 2} \\
13_{21 / 3}-474_{21 / 3} \\
13_{31 / 2}-474_{21 / 2}\end{array}$ & $(0.00)$ \\
\hline
\end{tabular}


TABLE 3.-Classified lines of W II-Continued

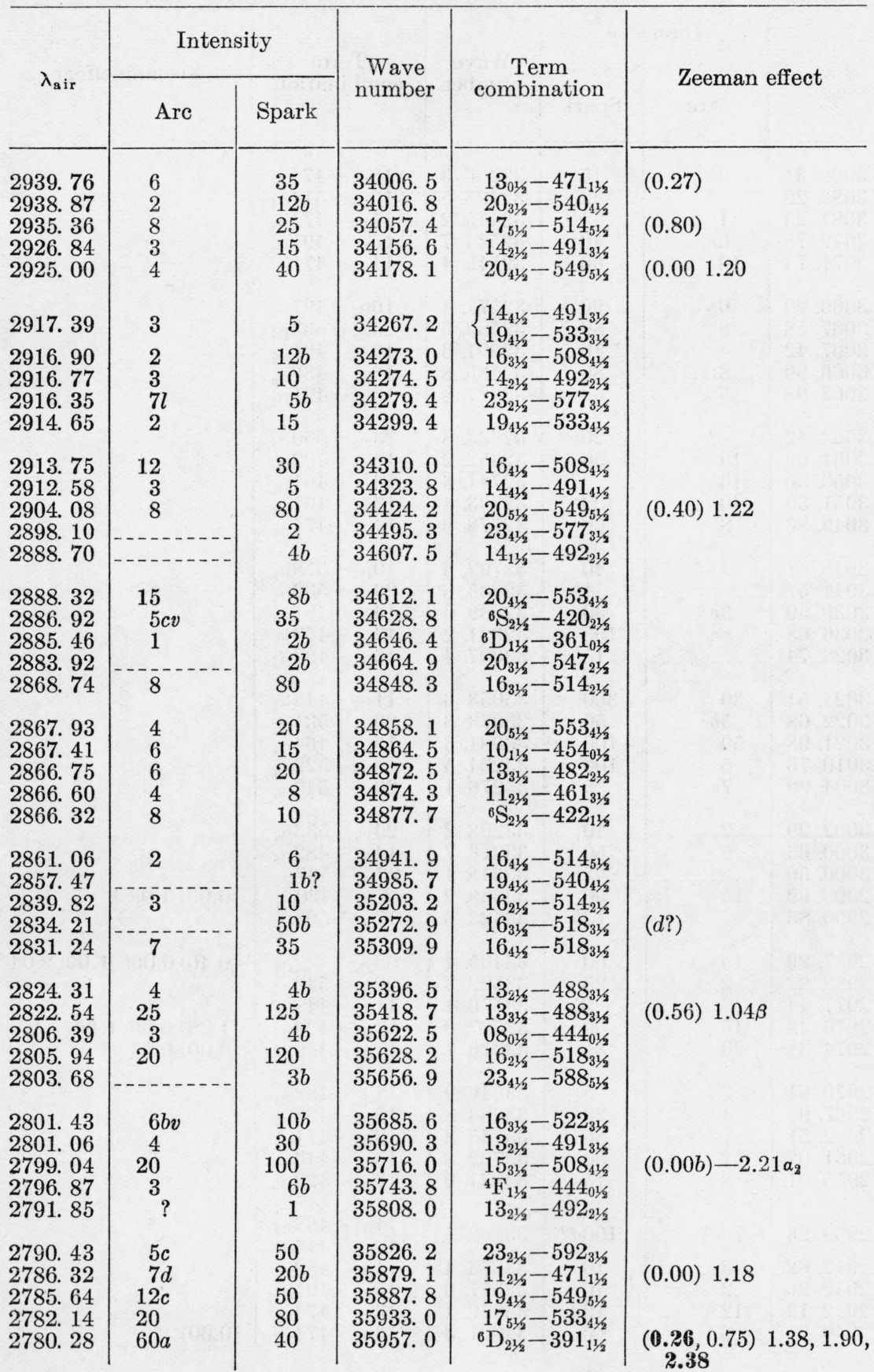


TABLE 3.-Classified lines of $\mathrm{W} \mathrm{II}-$ Continued

\begin{tabular}{|c|c|c|c|c|c|}
\hline \multirow{2}{*}{$\lambda_{\mathrm{air}}$} & \multicolumn{2}{|c|}{ Intensity } & \multirow{2}{*}{$\begin{array}{l}\text { Wave } \\
\text { number }\end{array}$} & \multirow{2}{*}{$\begin{array}{c}\text { Term } \\
\text { combination }\end{array}$} & \multirow{2}{*}{ Zeeman effect } \\
\hline & Arc & Spark & & & \\
\hline $\begin{array}{l}2776.51 \\
2773.85 \\
2768.32 \\
2764.26 \\
2761.59\end{array}$ & $\begin{array}{r}20 \\
5 \\
30 \\
200 \\
40\end{array}$ & $\begin{array}{c}100 \\
25 \\
50 \\
400 \\
100 b\end{array}$ & $\begin{array}{l}36005.8 \\
36040.4 \\
36112.3 \\
36165.4 \\
36200.4\end{array}$ & $\begin{array}{l}14_{41 / 2}-508_{41 / 3} \\
16_{21 / 2}-522_{31 / 2} \\
11_{21 / 3}-474_{21 / 2} \\
{ }^{6} \mathrm{D}_{01 / 2}-361_{01 / 3} \\
{ }^{4} \mathrm{~F}_{1 / 1 / 2}-449_{11 / 2}\end{array}$ & $\begin{array}{l}(0.16 ?) 1.22 \\
(0) 1.12 \\
(1.27) 1.93 \\
(0.88) 0.35,0.94,1.48\end{array}$ \\
\hline $\begin{array}{l}2758.33 \\
2754.70 \\
2753.10 \\
2752.36 \\
2750.33\end{array}$ & $\begin{array}{c}18 \\
1 \\
10\end{array}$ & $\begin{array}{c}20 \\
2 b \\
6 b \\
2 b \\
20\end{array}$ & $\begin{array}{l}36243.1 \\
36290.9 \\
36312.0 \\
36321.7 \\
36348.6\end{array}$ & $\begin{array}{l}{ }^{6} \mathrm{D}_{41 / 2}-423_{31 / 2} \\
15_{31 / 2}-514_{21 / 2} \\
16_{31 / 2}-529_{31 / 2} \\
19_{41 / 2}-553_{41 / 2} \\
16_{41 / 2}-529_{31 / 2}\end{array}$ & \\
\hline $\begin{array}{l}2745.04 \\
2737.13 \\
2732.38 \\
2729.62 \\
2728.88\end{array}$ & $\begin{array}{r}2 \\
1 \\
4 \\
25 \\
1\end{array}$ & $\begin{array}{c}30 b \\
3 b \\
4 b \\
75 \\
6 b\end{array}$ & $\begin{array}{l}36418.6 \\
36523.8 \\
36587.3 \\
36624.3 \\
36634.2\end{array}$ & $\begin{array}{l}23_{21 / 2}-598_{31 / 2} \\
16_{31 / 2}-531_{21 / 2} \\
10_{11 / 2}-471_{11 / 2} \\
08_{01 / 2}-454_{01 / 3} \\
23_{41 / 2}-598_{31 / 2}\end{array}$ & (0.91) 1.43 \\
\hline $\begin{array}{l}2726.44 \\
2722.80 \\
2721.85 \\
2720.60 \\
2720.40\end{array}$ & $\begin{array}{c}3 \\
20 \\
4 \\
25 d l \\
6\end{array}$ & $\begin{array}{r}4 \\
70 \\
15 \\
40 \\
30\end{array}$ & $\begin{array}{l}36667.0 \\
36716.0 \\
36728.8 \\
36745.7 \\
36748.4\end{array}$ & 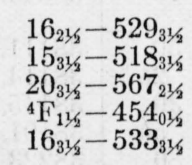 & $\begin{array}{l}(0.00) 0.89 b \\
(0.58)\end{array}$ \\
\hline $\begin{array}{l}2718.04 \\
2717.70 \\
2716.32 \\
2715.35 \\
2714.02\end{array}$ & $\begin{array}{r}30 \\
3 \\
30 \\
25\end{array}$ & $\begin{array}{c}120 \\
18 b \\
80 \\
80 \\
1 b\end{array}$ & $\begin{array}{l}36780.3 \\
36784.9 \\
36803.6 \\
36816.8 \\
36834.8\end{array}$ & $\begin{array}{l}16_{31 / 2}-533_{41 / 2} \\
16_{41 / 2}-533_{31 / 2} \\
14_{11 / 2}-514_{21 / 2} \\
16_{41 / 2}-533_{41 / 2} \\
20_{31 / 2}-568_{21 / 2}\end{array}$ & $\begin{array}{ll}(0.00) & 0.97 \\
& \\
(0.00) & 1.43 \\
(0.19) & 1.12\end{array}$ \\
\hline $\begin{array}{l}2710.79 \\
2709.58 \\
2706.73 \\
2705.60 \\
2703.12\end{array}$ & $\begin{array}{l}8 \\
20 \\
6 p v \\
10 b v \\
8\end{array}$ & $\begin{array}{l}40 \\
80 \\
50 b \\
35 b \\
10 ?\end{array}$ & $\begin{array}{l}36878.7 \\
36895.2 \\
36934.0 \\
36949.4 \\
36983.3\end{array}$ & $\begin{array}{l}16_{21 / 2}-531_{21 / 2} \\
14_{21 / 2}-518_{31 / 2} \\
{ }^{6} \mathrm{~S}_{21 / 2}-443_{21 / 2} \\
20_{41 / 2}-577_{31 / 2} \\
11_{21 / 2}-482_{21 / 2}\end{array}$ & $\begin{array}{l}(0.58) \\
(0.00)\end{array}$ \\
\hline $\begin{array}{l}2703.06 \\
2701.49 \\
2697.72 \\
2695.00 \\
2694.38\end{array}$ & $\begin{array}{r}8 \\
20 \\
80 \\
6 \\
20\end{array}$ & $\begin{array}{r}60 \\
60 \\
160 \\
30 \\
60\end{array}$ & $\begin{array}{l}36984.1 \\
37005.6 \\
37057.4 \\
37094.8 \\
37103.3\end{array}$ & $\begin{array}{r}23_{41 / 2}-602_{51 / 2} \\
14_{41 / 2}-518_{31 / 2} \\
{ }^{6} \mathrm{D}_{11 / 2}-385_{01 / 2} \\
16_{21 / 2}-533_{11 / 2} \\
16_{21 / 2}-533_{31 / 2}\end{array}$ & $\begin{array}{l}(0.00) 1.38 \\
(0.00) 2.08 a_{2} \\
\text { (b) } 1.94\end{array}$ \\
\hline $\begin{array}{l}2688.23 \\
2679.64 \\
2677.79 \\
2669.37 \\
2668.96\end{array}$ & $\begin{array}{c}7 \\
20 \\
20 \\
5 p \\
5\end{array}$ & $\begin{array}{l}35 \\
70 b \\
60 \\
30 \\
5 b\end{array}$ & $\begin{array}{l}37188.2 \\
37307.4 \\
37333.1 \\
37450.9 \\
37456.7\end{array}$ & $\begin{array}{l}16_{21 / 2}-534_{11 / 2} \\
14_{21 / 2}-522_{31 / 2} \\
{ }^{6} \mathrm{D}_{31 / 2}-420_{21 / 2} \\
13_{31 / 2}-508_{41 / 2} \\
{ }^{6} \mathrm{~S}_{21 / 2}-448_{31 / 2}\end{array}$ & $\begin{array}{l}(0.00) \\
(0.00)\end{array}$ \\
\hline $\begin{array}{l}2668.24 \\
2666.49 \\
2665.64 \\
2664.35 \\
2658.04\end{array}$ & $\begin{array}{l}1 \\
20 d ? \\
2 b \\
80 c v a \\
25\end{array}$ & $\begin{array}{r}5 b \\
60 \\
8 b \\
200 \\
100\end{array}$ & $\begin{array}{l}37466.8 \\
37491.3 \\
37503.3 \\
37521.5 \\
37610.6\end{array}$ & $\begin{array}{r}16_{31 / 2}-540_{41 / 2} \\
{ }^{6} \mathrm{~S}_{21 / 2}-449_{11 / 2} \\
16_{41 / 2}-540_{41 / 2} \\
17_{51 / 2}-549_{51 / 2} \\
{ }^{6} \mathrm{D}_{11 / 2}-391_{11 / 2}\end{array}$ & $\begin{array}{l}(0.41) 1.17 \beta \\
(0.40,1.08) 0.79,1.49 \text {, } \\
\quad 2.22\end{array}$ \\
\hline
\end{tabular}


TABLE 3.-Classified lines of $\mathrm{W}$ II-Continued

\begin{tabular}{|c|c|c|c|c|c|}
\hline \multirow{2}{*}{$\lambda_{\mathrm{air}}$} & \multicolumn{2}{|c|}{ Intensity } & \multirow{2}{*}{$\begin{array}{l}\text { Wave } \\
\text { number }\end{array}$} & \multirow{2}{*}{$\begin{array}{c}\text { Term } \\
\text { combination }\end{array}$} & \multirow{2}{*}{ Zeeman effect } \\
\hline & Arc & Spark & & & \\
\hline $\begin{array}{l}2655.67 \\
2653.57\end{array}$ & $\begin{array}{l}15 \\
20\end{array}$ & $\begin{array}{l}22 \\
35\end{array}$ & $\begin{array}{l}\text { 37644. } 1 \\
\text { 37673. } 9\end{array}$ & $\begin{array}{l}{ }^{4} \mathrm{~F}_{11 / 2}-463_{21 / 2} \\
{ }^{6} \mathrm{D}_{31 / 2}-423_{31 / 2}\end{array}$ & $(0.12 ?, \quad 0.57 ?, \quad 1.00$ \\
\hline $\begin{array}{l}2652.43 \\
2652.30 \\
2650.28\end{array}$ & $\begin{array}{l}1 \\
3\end{array}$ & $\begin{array}{l}4 b \\
3 b \\
7 b\end{array}$ & $\begin{array}{l}37690.1 \\
37691.9 \\
37720.6\end{array}$ & $\begin{array}{l}20_{31 / 2}-577_{31 / 2} \\
10_{11 / 2}-482_{21 / 2} \\
14_{11 / 2}-523_{01 / 2}\end{array}$ & \\
\hline $\begin{array}{l}2647.90 \\
2643.09 \\
2637.58 \\
2635.38 \\
2634.87\end{array}$ & $\begin{array}{c}1 p \\
? \\
15 \\
6 \\
20\end{array}$ & $\begin{array}{c}2 b \\
10 \\
25 \\
30 \\
9\end{array}$ & $\begin{array}{l}37754.6 \\
37823.3 \\
37902.3 \\
37933.9 \\
37941.3\end{array}$ & $\begin{array}{l}15_{31 / 2}-529_{31 / 2} \\
11_{21 / 2}-491_{31 / 2} \\
16_{21 / 3}-541_{11 / 2} \\
14_{21 / 3}-529_{31 / 2} \\
11_{21 / 2}-492_{21 / 2}\end{array}$ & \\
\hline $\begin{array}{l}2633.89 \\
2630.53 \\
2630.38 \\
2629.00 \\
2627.72\end{array}$ & $\begin{array}{l}4 \\
6 p \\
3 p \\
7 \\
4\end{array}$ & $\begin{array}{c}25 b \\
9 \\
20 \\
35 \\
10\end{array}$ & $\begin{array}{l}37955.4 \\
38003.8 \\
38006.0 \\
38026.0 \\
38044.5\end{array}$ & $\begin{array}{l}17_{51 / 2}-553_{41 / 2} \\
13_{21 / 3}-514_{21 / 2} \\
23_{41 / 2}-612_{51 / 3} \\
13_{31 / 3}-514_{21 / 2} \\
14_{41 / 2}-529_{31 / 3}\end{array}$ & $\begin{array}{l}(0.00) \\
(-) 0.94\end{array}$ \\
\hline $\begin{array}{l}2623.11 \\
2622.87 \\
2620.76 \\
2617.64 \\
2615.45\end{array}$ & $\begin{array}{c}6 p \\
1 p \\
7 p \\
5 l \\
20\end{array}$ & $\begin{array}{c}50 \\
2 b \\
30 \\
5 b \\
80\end{array}$ & $\begin{array}{l}38111.3 \\
38114.8 \\
38145.5 \\
38191.0 \\
38223.0\end{array}$ & $\begin{array}{l}20_{41 / 3}-588_{51 / 3} \\
16_{31 / 2}-547_{21 / 3} \\
14_{21 / 2}-531_{21 / 2} \\
15_{31 / 3}-533_{31 / 2} \\
15_{31 / 3}-533_{41 / 2}\end{array}$ & $\begin{array}{l}(0.00) 1.32 \\
(0.55) \\
(0.00) \quad 1.72 a_{2}\end{array}$ \\
\hline $\begin{array}{l}2606.97 \\
2606.47 \\
2606.27 \\
2605.97 \\
2605.41\end{array}$ & $\begin{array}{l}4 \\
4 \\
2 \\
7 \\
?\end{array}$ & $\begin{array}{l}12 \\
40 \\
20 b \\
15 \\
5 b\end{array}$ & $\begin{array}{l}38347.3 \\
38354.6 \\
38357.6 \\
38362.0 \\
38370.2\end{array}$ & $\begin{array}{l}08_{01 / 2}-471_{11 / 2} \\
23_{41 / 2}-615_{51 / 2} \\
20_{51 / 2}-588_{51 / 2} \\
14_{21 / 1}-533_{11 / 2} \\
14_{21 / 2}-533_{31 / 2}\end{array}$ & \\
\hline $\begin{array}{l}2603.02 \\
2601.43 \\
2599.65 \\
2598.75 \\
2598.67\end{array}$ & $\begin{array}{c}20 p \\
8 \\
3 \\
20 \\
7 ?\end{array}$ & $\begin{array}{c}120 \\
30 \\
7 \\
35 \\
7 ?\end{array}$ & $\begin{array}{l}38405.5 \\
38428.9 \\
38455.2 \\
38468.6 \\
38469.7\end{array}$ & $\begin{array}{l}16_{41 / 3}-549_{51 / 2} \\
13_{21 / 2}-518_{31 / 2} \\
14_{21 / 3}-534_{11 / 2} \\
4 \mathrm{~F}_{11 / 2}-471_{11 / 2} \\
16_{21 / 2}-547_{21 / 2}\end{array}$ & $\begin{array}{l}(0.00) 1.03 \\
(0.56) 0.42,0.8 \%, 1.25\end{array}$ \\
\hline $\begin{array}{l}2596.87 \\
2595.76 \\
2591.49 \\
2589.17 \\
2586.58\end{array}$ & $\begin{array}{r}3 \\
3 \\
14 \\
16 \\
1\end{array}$ & $\begin{array}{r}15 \\
8 \\
12 \\
90 \\
5\end{array}$ & $\begin{array}{l}38496.4 \\
38512.8 \\
38576.3 \\
38610.9 \\
38649.5\end{array}$ & $\begin{array}{l}20_{41 / 2}-592_{31 / 2} \\
14_{41 / 2}-533_{41 / 1} \\
{ }^{6} \mathrm{D}_{01 / 2}-385_{01 / 2} \\
{ }^{6} \mathrm{D}_{41 / 2}-447_{41 / 2} \\
10_{11 / 2}-492_{21 / 2}\end{array}$ & $\begin{array}{l}(0.00) 1.08 . \\
\left(1.13 \alpha_{2}\right) 1.46 \beta .\end{array}$ \\
\hline $\begin{array}{l}2585.93 \\
2583.52 \\
2581.20 \\
2579.56 \\
2577.31\end{array}$ & $\begin{array}{c}6 \\
3 p \\
18 \\
20 v\end{array}$ & $\begin{array}{c}30 \\
5 \\
30 \\
100 d \\
5 b\end{array}$ & $\begin{array}{l}\text { 38659. } 2 \\
38695.3 \\
38730.1 \\
38754.7 \\
38788.6\end{array}$ & $\begin{array}{l}19_{41 / 2}-577_{31 / 2} \\
14_{11 / 2}-533_{11 / 2} \\
{ }^{6} \mathrm{D}_{41 / 2}-448_{31 / 2} \\
{ }^{6} \mathrm{~S}_{21 / 2}-461_{31 / 2} \\
14_{11 / 2}-534_{11 / 2}\end{array}$ & \\
\hline $\begin{array}{l}2576.37 \\
2573.95 \\
2573.82 \\
2572.36 \\
2572.24\end{array}$ & $\begin{array}{r}8 \\
25 \\
3 \\
8 \\
8\end{array}$ & $\begin{array}{l}40 \\
7 \\
12 b \\
30 \\
45\end{array}$ & $\begin{array}{l}\text { 38802. } 7 \\
38839.2 \\
38841.1 \\
38863.2 \\
38865.0\end{array}$ & $\begin{array}{l}16_{31 / 2}-553_{41 / 2} \\
16_{41 / 2}-553_{41 / 2} \\
13_{21 / 2}-522_{31 / 2} \\
13_{31 / 2}-522_{31 / 2} \\
20_{51 / 2}-593_{41 / 3}\end{array}$ & $\begin{array}{l}(0.00) \\
(0.34) \\
(0.00) \\
1.23 .\end{array}$ \\
\hline
\end{tabular}


TABle 3.-Classified lines of W II-Continued

\begin{tabular}{|c|c|c|c|c|c|}
\hline \multirow{2}{*}{$\lambda_{\mathrm{air}}$} & \multicolumn{2}{|c|}{ Intensity } & \multirow{2}{*}{$\begin{array}{l}\text { Wave } \\
\text { number }\end{array}$} & \multirow{2}{*}{$\begin{array}{c}\text { Term } \\
\text { combination }\end{array}$} & \multirow{2}{*}{ Zeeman effect } \\
\hline & Arc & Spark & & & \\
\hline 2571. 46 & 40 & 150 & 38876.8 & ${ }^{6} \mathrm{D}_{21 / 2}-420_{21 / 2}$ & $\begin{array}{l}(0.84) 0.72, \mathbf{1 . 1 3}, \mathbf{1 . 5 0}, \\
\text { 1.86, 2.25. }\end{array}$ \\
\hline $\begin{array}{l}2569.30 \\
2567.62 \\
2557.51 \\
2555.10\end{array}$ & $\begin{array}{r}8 \\
10 \\
20\end{array}$ & $\begin{array}{r}80 \\
30 \\
2 \\
100\end{array}$ & $\begin{array}{l}38909.5 \\
38934.9 \\
39088.8 \\
39125.7\end{array}$ & $\begin{array}{l}15_{31 / 2}-540_{41 / 2} \\
{ }^{6} \mathrm{~S}_{21 / 2}-463_{21 / 2} \\
20_{41 / 2}-598_{31 / 2} \\
{ }^{6} \mathrm{D}_{21 / 2}-422_{11 / 2}\end{array}$ & $\begin{array}{l}(\mathbf{1 . 8 6 ,} 2.25 \\
(1.67) 1.33 . \\
(0.00) 1.82\end{array}$ \\
\hline $\begin{array}{l}2554.86 \\
2552.25 \\
2551.45 \\
2550.29 \\
2549.10\end{array}$ & \begin{tabular}{c}
$20 ?$ \\
5 \\
\hdashline $5 p$ \\
5
\end{tabular} & $\begin{array}{c}60 \\
5 \\
15 b \\
8 b \\
15\end{array}$ & $\begin{array}{l}\text { 39129. } 3 \\
39169.4 \\
39181.6 \\
39199.5 \\
39217.8\end{array}$ & $\begin{array}{l}{ }^{6} \mathrm{D}_{01 / 2}-391_{11 / 2} \\
14_{21 / 2}-541_{11 / 2} \\
13_{01 / 2}-523_{01 / 2} \\
14_{41 / 2}-540_{41 / 2} \\
{ }^{6} \mathrm{D}_{21 / 2}-423_{31 / 2}\end{array}$ & (1.04). \\
\hline $\begin{array}{l}2547.84 \\
2539.92 \\
2534.83 \\
2532.96 \\
2530.70\end{array}$ & $\begin{array}{r}3 \\
8 \\
6 \\
10\end{array}$ & $\begin{array}{c}5 b \\
20 b \\
50 \\
15 \\
8\end{array}$ & $\begin{array}{l}39237.2 \\
39359.5 \\
39438.5 \\
39467.6 \\
39502.9\end{array}$ & $\begin{array}{l}20_{31 / 2}-592_{31 / 2} \\
20_{31 / 2}-593_{41 / 2} \\
20_{41 / 2}-602_{51 / 2} \\
13_{21 / 2}-529_{31 / 2} \\
14_{11 / 2}-541_{11 / 2}\end{array}$ & $\begin{array}{l}(0.00) 1.32 . \\
(0.00) 1.16 . \\
(0.68) .\end{array}$ \\
\hline $\begin{array}{l}\text { 2527. } 20 \\
2526.21 \\
2522.04 \\
2519.44 \\
2519.12\end{array}$ & $\begin{array}{r}2 \\
4 \\
40 \\
8 \\
4\end{array}$ & $\begin{array}{r}15 \\
10 \\
80 \\
30 \\
7\end{array}$ & $\begin{array}{l}39557.6 \\
39573.1 \\
39638.5 \\
39679.4 \\
39684.5\end{array}$ & $\begin{array}{l}15_{31 / 2}-547_{21 / 2} \\
{ }^{4} \mathrm{~F}_{11 / 2}-482_{21 / 2} \\
{ }^{6} \mathrm{D}_{31 / 2}-443_{21 / 2} \\
13_{21 / 2}-531_{21 / 2} \\
20_{51 / 2}-602_{51 / 2}\end{array}$ & $\begin{array}{l}(0.00) . \\
(0.65) .\end{array}$ \\
\hline $\begin{array}{l}2515.81 \\
2514.36 \\
2510.48 \\
2509.95 \\
2508.69\end{array}$ & $\begin{array}{c}2 p \\
2 \\
15 \\
6 \\
?\end{array}$ & $\begin{array}{c}10 \\
4 b \\
75 \\
40 \\
4\end{array}$ & $\begin{array}{l}39736.7 \\
39759.6 \\
39821.0 \\
39829.4 \\
39849.4\end{array}$ & $\begin{array}{l}14_{21 / 2}-547_{21 / 2} \\
{ }^{6} \mathrm{~S}_{21 / 2}-471_{11 / 2} \\
19_{41 / 2}-588_{51 / 2} \\
20_{31 / 2}-598_{31 / 2} \\
16_{21 / 2}-560_{11 / 2}\end{array}$ & $\begin{array}{l}(0.45) \\
(0.00) 0.00 b, \mathbf{1 . 1 9}\end{array}$ \\
\hline $\begin{array}{l}\text { 2505. } 79 \\
2505.27 \\
2501.88 \\
2499.93 \\
2499.69\end{array}$ & $\begin{array}{c}3 p \\
30 l \\
3 \\
30 v\end{array}$ & $\begin{array}{c}5 \\
1 b \\
10 \\
4 \\
100\end{array}$ & $\begin{array}{l}\text { 39895. } 6 \\
\text { 39903. } 8 \\
39957.9 \\
39989.1 \\
39992.9\end{array}$ & $\begin{array}{l}13_{21 / 2}-533_{11 / 2} \\
13_{21 / 2}-533_{31 / 2} \\
13_{31 / 2}-533_{41 / 2} \\
13_{21 / 2}-534_{11 / 2} \\
{ }^{6} S_{21 / 2}-474_{21 / 2}\end{array}$ & $(1.23, \mathbf{2 . 0 3}) 0.00,0.71$, \\
\hline $\begin{array}{l}2497.48 \\
2496.65 \\
2494.87 \\
2492.93 \\
2490.72\end{array}$ & $\begin{array}{r}35 \\
50 \\
? \\
18 \\
12\end{array}$ & $\begin{array}{c}75 \\
120 l \\
7 \\
75 \\
25\end{array}$ & $\begin{array}{l}40028.3 \\
40041.6 \\
40070.2 \\
40101.4 \\
40136.9\end{array}$ & $\begin{array}{l}{ }^{6} \mathrm{D}_{41 / 2}-461_{31 / 2} \\
{ }^{6} \mathrm{D}_{31 / 2}-447_{11 / 2} \\
14_{11 / 2}-547_{21 / 2} \\
14_{41 / 2}-549_{51 / 2} \\
11_{21 / 2}-514_{21 / 2}\end{array}$ & $(0.50)$ \\
\hline $\begin{array}{l}2489.51 \\
2489.23 \\
2488.12 \\
2486.43 \\
2484.01\end{array}$ & $\begin{array}{l}5 \\
40 p r \\
8 p \\
6 p \\
7\end{array}$ & $\begin{array}{r}12 \\
200 \\
30 \\
18 \\
20\end{array}$ & $\begin{array}{l}40156.4 \\
40160.9 \\
40178.9 \\
40206.2 \\
40245.3\end{array}$ & $\begin{array}{l}13_{01 / 2}-533_{11 / 2} \\
{ }^{6} \mathrm{D}_{31 / 2}-448_{31 / 2} \\
16_{31 / 2}-567_{21 / 2} \\
19_{41 / 2}-592_{31 / 2} \\
15_{31 / 2}-553_{41 / 2}\end{array}$ & $\left(0.93 \alpha_{2}\right) 0.58 \ldots .2 .03$ \\
\hline $\begin{array}{l}2483.74 \\
2481.54 \\
2478.88 \\
2477.80 \\
2470.81\end{array}$ & $\begin{array}{c}5 \\
10 \\
4 p \\
25 \\
8\end{array}$ & $\begin{array}{r}7 \\
30 \\
10 \\
200 \\
70\end{array}$ & $\begin{array}{l}\text { 40249. } 7 \\
\text { 40285. } 4 \\
\text { 40328. } 6 \\
40346.2 \\
40460.3\end{array}$ & $\begin{array}{l}13_{01 / 2}-534_{11 / 2} \\
16_{31 / 2}-568_{21 / 2} \\
19_{41 / 2}-593_{41 / 2} \\
{ }^{6} \mathrm{D}_{41 / 2}-464_{41 / 2} \\
20_{41 / 2}-612_{51 / 2}\end{array}$ & $\left(0.88 \alpha_{2}\right) 1.39 \beta$ \\
\hline
\end{tabular}


TABLE 3.-Classified lines of W II-Continued

\begin{tabular}{|c|c|c|c|c|c|}
\hline \multirow{2}{*}{$\lambda_{\mathrm{air}}$} & \multicolumn{2}{|c|}{ Intensity } & \multirow{2}{*}{$\begin{array}{l}\text { Wave } \\
\text { number }\end{array}$} & \multirow{2}{*}{$\begin{array}{c}\text { Term } \\
\text { combination }\end{array}$} & \multirow{2}{*}{ Zeeman effect } \\
\hline & Arc & Spark & & & \\
\hline 2466. 52 & 35 & 80 & 40530.7 & ${ }^{6} \mathrm{D}_{13 / 5}-420_{21 / 2}$ & $(\mathbf{0 . 2 6 ,}, 0.81)$ 0.45, 0.99, \\
\hline $\begin{array}{l}2466.33 \\
2464.62 \\
2459.88 \\
2459.60\end{array}$ & $\begin{array}{c}5 p \\
14 \\
4 p \\
20\end{array}$ & $\begin{array}{l}10 \\
40 \\
30 \\
10\end{array}$ & $\begin{array}{l}40533.8 \\
40561.9 \\
40640.1 \\
40644.7\end{array}$ & $\begin{array}{l}16_{21 / 2}-567_{21 / 2} \\
11_{21 / 2}-518_{31 / 2} \\
16_{21 / 2}-568_{21 / 2} \\
13_{31 / 2}-540_{41 / 2}\end{array}$ & $\begin{array}{c}1.56 \\
(0.00) 0.73\end{array}$ \\
\hline $\begin{array}{l}2456.07 \\
2455.87 \\
2451.47 \\
2450.32 \\
2449.70\end{array}$ & $\begin{array}{c}4 p \\
6 \\
35 r l \\
2 \\
4\end{array}$ & $\begin{array}{l}8 \\
35 \\
50 d ? \\
7 \\
20\end{array}$ & $\begin{array}{l}40703.1 \\
40706.5 \\
40779.5 \\
40798.6 \\
40809.0\end{array}$ & $\begin{array}{l}13_{21 / 2}-541_{11 / 2} \\
20_{51 / 2}-612_{51 / 2} \\
{ }^{6} D_{11 / 2}-422_{11 / 2} \\
19_{41 / 2}-598_{31 / 2} \\
20_{43 / 2}-615_{51 / 2}\end{array}$ & $\left(0.49 \alpha_{2}\right) 1.49 \beta$ \\
\hline $\begin{array}{l}\text { 2447. } 52 \\
2446.39\end{array}$ & $25 v$ & $\begin{array}{r}1 \\
120\end{array}$ & $\begin{array}{l}\text { 40845. } 3 \\
40864.2\end{array}$ & $\begin{array}{l}10_{11 / 2}-514_{21 / 2} \\
{ }^{6} \mathrm{~S}_{21 / 2}-482_{21 / 2}\end{array}$ & $(0.80,1.40) 0.54,1.10$ \\
\hline $\begin{array}{l}2440.43 \\
2439.81 \\
2435.01\end{array}$ & $\begin{array}{r}10 \\
2 \\
10\end{array}$ & $\begin{array}{r}20 \\
7 \\
50\end{array}$ & $\begin{array}{l}\text { 40964. } 0 \\
40974.4 \\
\text { 41055. } 1\end{array}$ & $\begin{array}{l}12_{01 / 2}-541_{11 / 2} \\
11_{21 / 2}-522_{31 / 2} \\
20_{51 / 2}-615_{51 / 2}\end{array}$ & $(0.57)$ \\
\hline $\begin{array}{l}2431.37 \\
2429.97 \\
2429.53 \\
2427.81 \\
2427.49\end{array}$ & $\begin{array}{r}5 \\
1 \\
3 \\
4 \\
10\end{array}$ & $\begin{array}{r}18 \\
6 \\
8 \\
6 \\
40\end{array}$ & $\begin{array}{l}41116.6 \\
41140.3 \\
41147.7 \\
41176.9 \\
41182.3\end{array}$ & $\begin{array}{l}14_{21 / 2}-560_{11 / 2} \\
16_{31 / 2}-577_{31 / 2} \\
19_{41 / 2}-602_{51 / 2} \\
16_{41 / 2}-577_{31 / 2} \\
{ }^{6} D_{21 / 2}-443_{21 / 2}\end{array}$ & $(0.58) 1.55$ \\
\hline $\begin{array}{l}2422.29 \\
2420.99 \\
2414.13 \\
2411.82 \\
2411.54\end{array}$ & $\begin{array}{c}30 \\
12 \\
4 v \\
10 \\
6\end{array}$ & $\begin{array}{r}20 \\
35 \\
7 \\
25 \\
20\end{array}$ & $\begin{array}{l}41270.7 \\
41292.9 \\
41410.2 \\
41449.9 \\
41454.7\end{array}$ & $\begin{array}{l}13_{21 / 2}-547_{21 / 2} \\
13_{31 / 2}-547_{21 / 2} \\
6 \mathrm{~S}_{21 / 2}-488_{31 / 2} \\
14_{11 / 2}-560_{11 / 2} \\
17_{51 / 2}-588_{51 / 2}\end{array}$ & 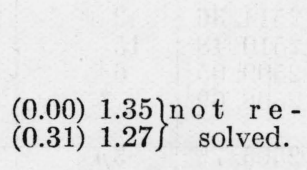 \\
\hline $\begin{array}{l}2411.29 \\
2403.07 \\
2401.86 \\
2400.86 \\
2397.10\end{array}$ & $\begin{array}{c}7 \\
5 \\
5 \\
4 \\
20 r\end{array}$ & $\begin{array}{r}3 \\
10 \\
12 \\
2 \\
200\end{array}$ & $\begin{array}{l}41459.0 \\
41600.8 \\
41621.7 \\
41639.0 \\
41704.4\end{array}$ & $\begin{array}{l}{ }^{6} \mathrm{D}_{31 / 2}-461_{31 / 2} \\
11_{21 / 2}-529_{31 / 2} \\
15_{31 / 2}-567_{21 / 2} \\
{ }^{6} \mathrm{D}_{31 / 2}-463_{21 / 2} \\
{ }^{6} \mathrm{D}_{21 / 2}-448_{31 / 2}\end{array}$ & $(0.00) 0.90$ \\
\hline $\begin{array}{l}2395.73 \\
2395.10 \\
2393.77 \\
2392.93\end{array}$ & $\begin{array}{r}5 \\
9 \\
1 \\
20\end{array}$ & $\begin{array}{r}8 l \\
10 \\
3 \\
60\end{array}$ & $\begin{array}{l}41728.2 \\
41739.2 \\
41762.4 \\
41777.0\end{array}$ & $\begin{array}{l}15_{31 / 2}-568_{21 / 2} \\
{ }^{6} \mathrm{D}_{21 / 2}-449_{11 / 2} \\
10_{11 / 2}-523_{01 / 2} \\
{ }^{6} \mathrm{D}_{31 / 2}-464_{41 / 2}\end{array}$ & $\left(\begin{array}{lll}0.00, & 0.61 ?, & 0.89 ?)\end{array}\right.$ \\
\hline 2391. 59 & $2 v$ & 3 & 41800.4 & $14_{21 / 2}-567_{21 / 2}$ & \\
\hline $\begin{array}{l}2390.89 \\
2390.37 \\
2385.50 \\
2382.36 \\
2381.33\end{array}$ & $\begin{array}{r}4 \\
25 \\
8 \\
7 \\
6\end{array}$ & $\begin{array}{r}30 \\
75 \\
12 \\
20 \\
8\end{array}$ & $\begin{array}{l}41812.7 \\
41821.8 \\
41907.1 \\
41962.4 \\
41980.5\end{array}$ & $\begin{array}{l}11_{21 / 2}-531_{21 / 2} \\
{ }^{6} \mathrm{~S}_{21 / 2}-492_{21 / 2} \\
14_{21 / 2}-568_{21 / 2} \\
17_{51 / 2}-593_{41 / 2} \\
13_{31 / 2}-553_{41 / 2}\end{array}$ & $\begin{array}{l}(0.00) 1.11 \\
(0.50 ?, 0.9 \gamma) 1.72 \beta \\
(0.00)\end{array}$ \\
\hline $\begin{array}{l}2378.60 \\
2378.13 \\
2373.31 \\
2370.62 \\
2366.68\end{array}$ & \begin{tabular}{c}
10 \\
8 \\
\hdashline 5 \\
$4 l$
\end{tabular} & $\begin{array}{r}15 \\
15 \\
1 \\
12 \\
6\end{array}$ & $\begin{array}{l}42028.7 \\
42037.0 \\
42122.4 \\
42170.2 \\
42240.4\end{array}$ & $\begin{array}{l}11_{21 / 2}-533_{11 / 2} \\
11_{21 / 5}-533_{31 / 2} \\
11_{21 / 1}-534_{11 / 5} \\
19_{41 / 3}-612_{51 / 2} \\
14_{11 / 2}-568_{21 / 2}\end{array}$ & \\
\hline
\end{tabular}


TABLE 3.-Classified lines of W II-Continued

\begin{tabular}{|c|c|c|c|c|c|}
\hline \multirow{2}{*}{$\lambda_{\text {air }}$} & \multicolumn{2}{|c|}{ Intensity } & \multirow{2}{*}{$\begin{array}{l}\text { Wave } \\
\text { number }\end{array}$} & \multirow{2}{*}{$\begin{array}{c}\text { Term } \\
\text { combination }\end{array}$} & \multirow{2}{*}{ Zeeman effect } \\
\hline & Arc & Spark & & & \\
\hline $\begin{array}{l}\text { 2363. } 46 \\
2361.19 \\
2351.17 \\
2351.05 \\
2347.64\end{array}$ & $\begin{array}{r}8 \\
12 \\
1 \\
2 \\
1\end{array}$ & $\begin{array}{c}14 \\
10 \\
1 b \\
6 \\
3 b\end{array}$ & $\begin{array}{l}42297.8 \\
42338.6 \\
42519.0 \\
42521.2 \\
42582.8\end{array}$ & $\begin{array}{l}{ }^{6} \mathrm{D}_{01 / 2}-422_{11 / 5} \\
16_{41 / 2}-588_{51 / 2} \\
19_{41 / 2}-615_{51 / 2} \\
10_{11 / 2}-531_{21 / 2} \\
15_{31 / 2}-577_{31 / 2}\end{array}$ & $(0.00) 1.24$ \\
\hline $\begin{array}{l}2341.90 \\
2341.37\end{array}$ & $\begin{array}{r}1 \\
25\end{array}$ & $\begin{array}{r}6 \\
35\end{array}$ & $\begin{array}{l}42687.3 \\
42696.9\end{array}$ & $\begin{array}{l}16_{31 / 2}-592_{31 / 2} \\
{ }^{6} \mathrm{D}_{31 / 2}-474_{21 / 2}\end{array}$ & $\begin{array}{c}(0.22, \mathbf{0 . 6 8}, \quad 1.15) \\
0.42 ?, 2.07, \mathbf{2 . 5 1}\end{array}$ \\
\hline $\begin{array}{l}2339.90 \\
2339.73 \\
2339.16\end{array}$ & $\begin{array}{r}6 \\
10 \\
15\end{array}$ & $\begin{array}{r}20 \\
8 \\
30\end{array}$ & $\begin{array}{l}42723.8 \\
42726.9 \\
42737.3\end{array}$ & $\begin{array}{l}16_{43 / 3}-592_{31 / 2} \\
{ }^{4} \mathrm{~F}_{11 / 2}-514_{21 / 2} \\
10_{11 / 2}-533_{1 / 2}\end{array}$ & $(0.00) 1.25$ \\
\hline $\begin{array}{l}\text { 2337. } 80 \\
2336.71 \\
2335.21 \\
2334.07 \\
2333.77\end{array}$ & $\begin{array}{r}6 \\
6 \\
6 \\
5 \\
15\end{array}$ & $\begin{array}{r}20 \\
15 \\
25 \\
5 \\
35\end{array}$ & $\begin{array}{l}42762.1 \\
42781.9 \\
42809.6 \\
42830.5 \\
42836.0\end{array}$ & $\begin{array}{l}14_{21 / 2}-577_{31 / 2} \\
17_{51 / 2}-602_{51 / 2} \\
16_{31 / 2}-593_{41 / 2} \\
10_{11 / 2}-534_{11 / 2} \\
{ }^{6} D_{11 / 2}-443_{21 / 2}\end{array}$ & $(0.25,0.75) 0.71,1.31$ \\
\hline $\begin{array}{l}\text { 2331. } 78 \\
2329.69 \\
2328.32 \\
2326.09 \\
2324.71\end{array}$ & $\begin{array}{l}3 \\
6 \\
20 \\
15 r ? \\
1\end{array}$ & $\begin{array}{r}4 \\
15 \\
35 \\
60 \\
1\end{array}$ & $\begin{array}{l}42872.5 \\
42911.0 \\
42936.2 \\
42977.4 \\
43002.8\end{array}$ & $\begin{array}{l}14_{41 / 2}-577_{31 / 2} \\
13_{01 / 2}-560_{11 / 2} \\
{ }^{6} \mathrm{D}_{11 / 2}-444_{01 / 2} \\
{ }^{6} \mathrm{D}_{41 / 2}-491_{31 / 2} \\
{ }^{6} \mathrm{D}_{21 / 2}-461_{31 / 2}\end{array}$ & $\begin{array}{l}(0.34) 0.61,1.86 \\
(1.05) 2.91 \\
(0.00) 1.62\end{array}$ \\
\hline $\begin{array}{l}2323.04 \\
2322.59 \\
2315.02\end{array}$ & $\begin{array}{r}15 \\
2 \\
20\end{array}$ & $\begin{array}{c}25 \\
3 b \\
50\end{array}$ & $\begin{array}{l}43033.8 \\
43042.2 \\
43182.9\end{array}$ & $\begin{array}{l}{ }^{6} \mathrm{D}_{41 / 2}-491_{41 / 2} \\
16_{21 / 2}-592_{31 / 2} \\
{ }^{6} \mathrm{D}_{21 / 2}-463_{21 / 2}\end{array}$ & $\begin{array}{l}(0.38) 1.14 \\
(0.561 t 05) 0.81, \mathbf{1 . 4 0}\end{array}$ \\
\hline $\begin{array}{l}2309.85 \\
2307.93\end{array}$ & $\begin{array}{l}8 \\
5\end{array}$ & $\begin{array}{l}25 \\
10\end{array}$ & $\begin{array}{l}43279.5 \\
43315.5\end{array}$ & $\begin{array}{l}16_{31 / 2}-598_{31 / 2} \\
16_{41 / 2}-598_{31 / 2}\end{array}$ & \\
\hline $\begin{array}{l}\text { 2306. } 92 \\
2303.82\end{array}$ & $\begin{array}{l}15 \\
25\end{array}$ & $\begin{array}{l}40 \\
75\end{array}$ & $\begin{array}{l}43334.5 \\
43392.8\end{array}$ & $\begin{array}{l}13_{21 / 2}-567_{21 / 2} \\
{ }^{6} \mathrm{D}_{11 / 2}-449_{11 / 2}\end{array}$ & $\begin{array}{c}(0.34, \quad 0.99) \\
1.5 \%, 2.24\end{array}$ \\
\hline $\begin{array}{l}\text { 2301. } 28 \\
2300.08 \\
2296.96\end{array}$ & $\begin{array}{l}3 \\
1 \\
8\end{array}$ & $\begin{array}{l}5 \\
5 \\
6\end{array}$ & $\begin{array}{l}\text { 43440. } 7 \\
\text { 43463. } 3 \\
43522.4\end{array}$ & $\begin{array}{l}13_{21 / 2}-568_{21 / 2} \\
13_{31 / 2}-568_{21 / 2} \\
08_{01 / 2}-523_{01 / 2}\end{array}$ & \\
\hline $\begin{array}{l}2295.78 \\
2294.55 \\
2290.56 \\
2289.41 \\
2282.20\end{array}$ & $\begin{array}{l}15 \\
10 ? \\
15 \\
5 \\
25\end{array}$ & $\begin{array}{r}20 \\
35 \\
20 \\
7 \\
75\end{array}$ & $\begin{array}{l}43544.7 \\
43568.1 \\
43644.0 \\
43665.8 \\
43803.8\end{array}$ & $\begin{array}{l}10_{11 / 2}-541_{11 / 2} \\
{ }^{6} D_{31 / 2}-482_{21 / 2} \\
{ }^{4} \mathrm{~F}_{11 / 2}-523_{01 / 2} \\
16_{41 / 2}-602_{51 / 2} \\
17_{51 / 2}-612_{51 / 2}\end{array}$ & $(0.35) 1.16$ \\
\hline $\begin{array}{l}2275.22 \\
2271.64 \\
2271.10 \\
2270.23 \\
2266.25\end{array}$ & $\begin{array}{r}5 \\
2 \\
10 \\
25 \\
15\end{array}$ & $\begin{array}{r}12 \\
125 \\
80\end{array}$ & $\begin{array}{l}43938.2 \\
44007.4 \\
44017.7 \\
44034.8 \\
44112.1\end{array}$ & $\begin{array}{l}{ }^{6} \mathrm{D}_{11 / 2}-454_{01 / 2} \\
{ }^{6} \mathrm{D}_{21 / 2}-471_{11 / 2} \\
{ }^{6} \mathrm{~S}_{21 / 2}-514_{21 / 2} \\
14_{41 / 2}-588_{51 / 2} \\
10_{11 / 2}-547_{21 / 2}\end{array}$ & \\
\hline $\begin{array}{l}2266.12 \\
2265.34 \\
2264.18 \\
2259.66 \\
2259.07\end{array}$ & $\begin{array}{r}15 \\
7 \\
8 \\
5 \\
10\end{array}$ & $\begin{array}{r}80 \\
35 \\
35 \\
3 \\
25\end{array}$ & $\begin{array}{l}44114.6 \\
44129.8 \\
44152.4 \\
44240.7 \\
44252.3\end{array}$ & $\begin{array}{l}{ }^{6} \mathrm{D}_{31 / 2}-488_{31 / 2} \\
15_{31 / 2}-592_{31 / 2} \\
17_{51 / 2}-615_{51 / 2} \\
{ }^{6} \mathrm{D}_{21 / 2}-474_{21 / 2} \\
15_{31 / 2}-593_{41 / 2}\end{array}$ & $(\ldots \ldots 1.40,1.92) ?$ \\
\hline
\end{tabular}


TABLE 3.-Classified lines of $\mathrm{W} \mathrm{II}-$ Continued

\begin{tabular}{|c|c|c|c|c|c|}
\hline \multirow{2}{*}{$\lambda_{\text {air }}$} & \multicolumn{2}{|c|}{ Intensity } & \multirow{2}{*}{$\begin{array}{l}\text { Wave } \\
\text { number }\end{array}$} & \multirow{2}{*}{$\begin{array}{c}\text { Term } \\
\text { combination }\end{array}$} & \multirow{2}{*}{ Zeeman effect } \\
\hline & Arc & Spark & & & \\
\hline $\begin{array}{l}2256.85 \\
2256.18 \\
2255.71 \\
2251.43 \\
2251.14\end{array}$ & $\begin{array}{r}12 \\
2 \\
10 \\
18 \\
30\end{array}$ & $\begin{array}{r}30 \\
6 \\
10 \\
25 \\
40\end{array}$ & $\begin{array}{l}44295.8 \\
44308.9 \\
44318.1 \\
44402.4 \\
44408.1\end{array}$ & $\begin{array}{l}13_{21 / 2}-577_{31 / 5} \\
14_{21 / 2}-592_{31 / 2} \\
1_{31 / 5}-577_{31 / 2} \\
{ }^{4} \mathrm{~F}_{11 / 2}-531_{21 / 2} \\
{ }^{6} \mathrm{D}_{31 / 2}-491_{31 / 2}\end{array}$ & \\
\hline $\begin{array}{l}2250.56 \\
2249.38 \\
2248.75 \\
2248.27 \\
2246.64\end{array}$ & $\begin{array}{l}3 \\
15 \\
60 r \\
25 v \\
15\end{array}$ & $\begin{array}{r}3 \\
12 \\
100 \\
40 \\
20\end{array}$ & $\begin{array}{l}44419.6 \\
44442.9 \\
44455.3 \\
44464.8 \\
44497.1\end{array}$ & $\begin{array}{r}14_{41 / 6}-592_{31 / 2} \\
{ }^{6} \mathrm{~S}_{21 / 2}-518_{31 / 2} \\
{ }^{6} \mathrm{D}_{01 / 2}-444_{01 / 6} \\
{ }^{6} \mathrm{D}_{31 / 2}-491_{41 / 6} \\
08_{01 / 2}-533_{11 / 2}\end{array}$ & $\begin{array}{l}(0.00) \\
(1.68) \\
(0.00)\end{array}$ \\
\hline $\begin{array}{l}2245.19 \\
2244.42 \\
2240.53 \\
2237.06 \\
2235.85\end{array}$ & $\begin{array}{c}25 \\
1 \\
2 ? \\
15 \\
8\end{array}$ & $\begin{array}{r}40 l \\
3 \\
100 \\
8\end{array}$ & $\begin{array}{l}44525.8 \\
44541.1 \\
44618.5 \\
44687.6 \\
44711.7\end{array}$ & $\begin{array}{l}{ }^{6} \mathrm{D}_{31 / 5}-492_{21 / 2} \\
14_{41 / 2}-593_{41 / 5} \\
{ }^{4} \mathrm{~F}_{1 / 5}-533_{11 / 5} \\
16_{41 / 2}-612_{51 / 2} \\
{ }^{4} \mathrm{~F}_{11 / 2}-534_{1 / 2}\end{array}$ & $\begin{array}{l}(0.00) 1.68 \\
(0.00) 1.08\end{array}$ \\
\hline $\begin{array}{l}2235.64 \\
2235.37 \\
2232.28 \\
2229.62 \\
2228.70\end{array}$ & $\begin{array}{r}20 \\
6 \\
6 \\
75 \\
5\end{array}$ & $\begin{array}{r}30 \\
60 \\
4 \\
100 \\
2 b\end{array}$ & $\begin{array}{l}44716.0 \\
44721.4 \\
44783.3 \\
44836.6 \\
44855.1\end{array}$ & $\begin{array}{l}{ }^{6} \mathrm{D}_{41 / 2}-508_{41 / 2} \\
15_{31 / 2}-598_{31 / 2} \\
{ }^{1} 1_{21 / 2}-560_{11 / 2} \\
{ }^{6} \mathrm{D}_{11 / 2}-463_{21 / 2} \\
{ }^{6} \mathrm{~S}_{21 / 2}-522_{31 / 2}\end{array}$ & \\
\hline $\begin{array}{l}2226.40 \\
2225.88 \\
2220.94 \\
2219.74 \\
2216.03\end{array}$ & $\begin{array}{r}8 \\
120 \\
40 \\
15 \\
60\end{array}$ & $\begin{array}{r}15 \\
150 \\
100 \\
12 \\
40\end{array}$ & $\begin{array}{l}44901.6 \\
44912.0 \\
45011.9 \\
45036.3 \\
45111.7\end{array}$ & $\begin{array}{l}14_{21 / 2}-598_{31 / 2} \\
{ }^{6} \mathrm{D}_{01 / 2}-449_{11 / 2} \\
14_{41 / 2}-598_{31 / 2} \\
16_{41 / 2}-615_{51 / 2} \\
{ }^{6} \mathrm{D}_{21 / 2}-482_{21 / 2}\end{array}$ & $\begin{array}{l}(0.00) \\
(1.00) 0.24,2.24 \\
(0.00) \\
(0.65) 1.43\end{array}$ \\
\hline $\begin{array}{l}2206.60 \\
2204.49 \\
2203.80 \\
2200.70 \\
2199.17\end{array}$ & $\begin{array}{r}40 \\
200 \\
10 \\
2 \\
40\end{array}$ & $\begin{array}{r}200 \\
300 \\
25 \\
1 \\
2\end{array}$ & $\begin{array}{l}45304.4 \\
45347.8 \\
45362.0 \\
45425.9 \\
45457.4\end{array}$ & $\begin{array}{l}08_{01 / /}-541_{11 / 2} \\
{ }^{6} \mathrm{D}_{41 / 2}-514_{51 / 2} \\
14_{41 / 2}-602_{51 / 3} \\
{ }^{4} \mathrm{~F}_{11 / /}-541_{11 / 2} \\
{ }^{6} \mathrm{D}_{01 / 2}-454_{01 / 2}\end{array}$ & $(0.00) 0.96$ \\
\hline $\begin{array}{l}2198.68 \\
2198.00 \\
2197.50 \\
2193.54 \\
2189.50\end{array}$ & $\begin{array}{l}50 \\
8 \\
8 \\
30 d \\
80\end{array}$ & $\begin{array}{r}80 \\
6 \\
10 \\
40 \\
50\end{array}$ & $\begin{array}{l}45467.6 \\
45481.6 \\
45492.0 \\
45574.1 \\
45658.3\end{array}$ & $\begin{array}{l}11_{21 / 2}-567_{21 / 2} \\
{ }^{6} \mathrm{~S}_{21 / 2}-529_{31 / 2} \\
10_{11 / 2}-560_{11 / 2} \\
11_{21 / 2}-568_{21 / 2} \\
{ }^{6} \mathrm{D}_{21 / 2}-488_{31 / 2}\end{array}$ & \\
\hline $\begin{array}{l}2189.36 \\
2187.82 \\
2186.73 \\
2180.70 \\
2179.64\end{array}$ & $\begin{array}{c}50 \\
3 \\
80 v \\
10 \\
8\end{array}$ & $\begin{array}{c}40 \\
1 l \\
40 \\
8 \\
8\end{array}$ & $\begin{array}{l}45661.1 \\
45693.3 \\
45716.0 \\
45842.5 \\
45864.7\end{array}$ & $\begin{array}{l}{ }^{6} \mathrm{D}_{11 / 2}-471_{11 / 2} \\
{ }^{6} \mathrm{~S}_{21 / 2}-531_{21 / 2} \\
{ }^{6} \mathrm{D}_{41 / 2}-518_{31 / 2} \\
13_{21 / 2}-592_{31 / 2} \\
13_{31 / 2}-592_{31 / 2}\end{array}$ & \\
\hline $\begin{array}{l}2178.23 \\
2177.56 \\
2177.13 \\
2175.49 \\
2173.83\end{array}$ & $\begin{array}{l}2 \\
40 r ? v \\
3 \\
12 \\
15\end{array}$ & $\begin{array}{r}30 \\
2 \\
10\end{array}$ & $\begin{array}{l}45894.4 \\
45908.5 \\
45917.5 \\
45952.1 \\
45987.4\end{array}$ & $\begin{array}{l}{ }^{6} \mathrm{D}_{11 / 2}-474_{21 / 2} \\
{ }^{6} \mathrm{~S}_{21 / 2}-533_{11 / 2} \\
{ }^{6} \mathrm{~S}_{21 / 2}-533_{31 / 2} \\
{ }^{6} \mathrm{D}_{21 / 2}-491_{31 / 2} \\
{ }^{1} 3_{31 / 2}-593_{41 / 2}\end{array}$ & \\
\hline $\begin{array}{l}2173.55 \\
2169.95 \\
2167.19 \\
2166.32 \\
2159.96\end{array}$ & $\begin{array}{l}60 \\
50 \\
12 \\
80 r ? \\
40\end{array}$ & $\begin{array}{r}70 \\
12 \\
2 \\
80 \\
3\end{array}$ & $\begin{array}{l}45993.2 \\
46069.5 \\
46128.2 \\
46146.8 \\
46282.6\end{array}$ & $\begin{array}{l}{ }^{4} \mathrm{~F}_{11 / 2}-5477_{21 / 5} \\
{ }^{6} \mathrm{D}_{21 / 2}-492_{21 / 5} \\
{ }^{6} \mathrm{D}_{41 / 5}-522_{31 / 5} \\
{ }^{6} \mathrm{D}_{31 / 5}-508_{41 / 6} \\
{ }^{10} 0_{13 / 5}-568_{21 / 5}\end{array}$ & \\
\hline
\end{tabular}


TABLE 3.-Classified lines of W II-Continued

\begin{tabular}{|c|c|c|c|c|c|}
\hline \multirow{2}{*}{$\lambda_{\mathrm{air}}$} & \multicolumn{2}{|c|}{ Intensity } & \multirow{2}{*}{$\begin{array}{c}\text { Wave } \\
\text { number }\end{array}$} & \multirow{2}{*}{$\begin{array}{c}\text { Term } \\
\text { combination }\end{array}$} & \multirow{2}{*}{ Zeeman effect } \\
\hline & Arc & Spark & & & \\
\hline $\begin{array}{l}2155.25 \\
2153.15 \\
2151.83 \\
2139.64 \\
2139.16\end{array}$ & $\begin{array}{r}3 \\
8 \\
6 \\
12 \\
30\end{array}$ & $\begin{array}{c}1 \\
4\end{array}$ & $\begin{array}{l}46383.7 \\
46429.0 \\
46457.3 \\
46722.0 \\
46732.5\end{array}$ & $\begin{array}{l}14_{41 / 3}-612_{51 / 2} \\
11_{21 / 4}-577_{31 / 2} \\
13_{31 / 2} 598_{31 / 2} \\
{ }^{6} \mathrm{D}_{31 / 2}-514_{21 / 2} \\
14_{41 / 2}-615_{51 / 2}\end{array}$ & $8+\frac{1}{80}$ \\
\hline $\begin{array}{l}2138.14 \\
2137.64 \\
2120.37 \\
2118.87 \\
2118.34\end{array}$ & $\begin{array}{r}50 \\
40 \\
1 \\
70 \\
2\end{array}$ & $\begin{array}{r}30 \\
5 \\
-12\end{array}$ & $\begin{array}{l}46754.8 \\
46765.8 \\
47146.6 \\
47180.0 \\
47191.8\end{array}$ & $\begin{array}{l}{ }^{6} \mathrm{D}_{41 / 3}-529_{31 / 3} \\
{ }^{6} \mathrm{D}_{11 / 3}-482_{21 / 3} \\
{ }^{6} \mathrm{D}_{31 / 3}-518_{31 / 3} \\
{ }^{6} \mathrm{D}_{01 / 1}-471_{11 / 2} \\
{ }^{6} \mathrm{D}_{41 / 2}-533_{31 / 2}\end{array}$ & \\
\hline $\begin{array}{l}2116.94 \\
2115.66 \\
2101.96 \\
2094.72 \\
2086.58\end{array}$ & $\begin{array}{r}40 \\
2 \\
20 \\
120 \\
5\end{array}$ & 2 & $\begin{array}{l}47223.0 \\
47251.6 \\
47559.5 \\
47723.8 \\
47910.0\end{array}$ & $\begin{array}{c}{ }^{6} \mathrm{D}_{41 / 1}-533_{41 / 2} \\
08_{01 / 2}-560_{11 / 2} \\
{ }^{6} \mathrm{D}_{31 / 3}-522_{31 / 3} \\
{ }^{6} \mathrm{D}_{11 / 3}-492_{21 / 3} \\
{ }^{6} \mathrm{D}_{41 / 2}-540_{41 / 3}\end{array}$ & \\
\hline $\begin{array}{l}2083.70 \\
2074.64 \\
2071.22 \\
2065.58 \\
2058.30\end{array}$ & $\begin{array}{l}10 \\
20 \\
30 \\
20 r ? \\
12\end{array}$ & $\begin{array}{c}1 \\
10 \\
20 b\end{array}$ & $\begin{array}{l}\text { 47976. } 2 \\
48185.7 \\
48265.3 \\
48397.0 \\
48568.2\end{array}$ & $\begin{array}{l}{ }^{1} 1_{21 / 3}-592_{31 / 2} \\
{ }^{6} \mathrm{D}_{33 / 2}-529_{31 / 2} \\
{ }^{6} \mathrm{D}_{21 / 3}-514_{21 / 2} \\
{ }^{6} \mathrm{D}_{31 / 3}-531_{21 / 2} \\
11_{21 / 2}-598_{31 / 6}\end{array}$ & \\
\hline $\begin{array}{l}2056.01 \\
2054.66 \\
2053.11 \\
2048.04 \\
2035.87\end{array}$ & $\begin{array}{c}2 \\
15 \\
6 b \\
5 \\
5\end{array}$ & $\begin{array}{c}6 \\
2 \\
8 \\
50 b \\
40 b\end{array}$ & $\begin{array}{l}48622.2 \\
48654.2 \\
48690.9 \\
48811.4 \\
49103.2\end{array}$ & $\begin{array}{l}{ }^{6} \mathrm{D}_{31 / 2}-533_{31 / 3} \\
{ }^{6} \mathrm{D}_{31 / 3}-533_{41 / 2} \\
{ }^{6} \mathrm{D}_{21 / 2}-518_{31 / 3} \\
{ }^{6} \mathrm{D}_{41 / 3}-549_{51 / 3} \\
{ }^{6} \mathrm{D}_{21 / 2}-522_{31 / 2}\end{array}$ & \\
\hline $\begin{array}{l}\text { 2029. } 99 \\
2026.06 \\
2021.43 \\
2010.21 \\
2002.56\end{array}$ & $\begin{array}{l}5 \\
8 \\
-3\end{array}$ & $\begin{array}{l}20 \\
30 v \\
1 b \\
8 \\
1\end{array}$ & $\begin{array}{l}49245.4 \\
49340.9 \\
49453.9 \\
49729.9 \\
49919.8\end{array}$ & $\begin{array}{c}{ }^{6} \mathrm{D}_{41 / 1}-553_{41 / 2} \\
{ }^{6} \mathrm{D}_{31 / 2}-540_{41 / 2} \\
{ }^{6} \mathrm{~S}_{21 / 3}-568_{21 / 2} \\
{ }^{6} \mathrm{D}_{21 / 3}-529_{31 / 2} \\
{ }^{6} \mathrm{D}_{11 / 2}-514_{21 / 2}\end{array}$ & \\
\hline $\begin{array}{l}\text { 2001. } 70 \\
\text { 1999. } 82 \\
1989.40 \\
1972.63 \\
1961.43\end{array}$ & $\begin{array}{l}3 \\
3 \\
2 \\
2 \\
2\end{array}$ & $\begin{array}{l}8 \\
5 \\
5 \\
4 \\
5\end{array}$ & $\begin{array}{l}49941.3 \\
49988.2 \\
50250.0 \\
50677.1 \\
50966.4\end{array}$ & $\begin{array}{l}{ }^{6} \mathrm{D}_{21 / 2}-531_{21 / 2} \\
{ }^{6} \mathrm{D}_{31 / 2}-547_{21 / 2} \\
{ }^{6} \mathrm{D}_{21 / 3}-534_{11 / 2} \\
{ }^{6} \mathrm{D}_{31 / 2}-553_{41 / 2} \\
{ }^{6} \mathrm{D}_{21 / 2}-541_{11 / 2}\end{array}$ & \\
\hline
\end{tabular}

The observed Zeeman effects in the last column of table 3 are those measured on recent Bureau plates; or are taken from Jack ${ }^{7}$ or Beining, ${ }^{8}$ in which case they are followed by the letters $\mathbf{J}$ or Be, respectively. Jack's observations have been reduced by 7 percent and Beining's have been corrected according to Catalán and Poggio. ${ }^{9}$ Typical unresolved patterns have been described by Greek letters as follows: Doublet shaded outward, $\alpha_{1}$; doublet shaded inward, $\alpha_{2}$; doublet shaded symmetrically, $\beta$. The observed patterns of table 3 may be compared with the theoretical patterns derived from the

\footnotetext{
T R. Jack, Ann. Physik [4] 28, 1032 (1909).

8 H. Beining, Z. Physik 42, 153 (1927).

M. A. Catalán and F. Poggio, Anales. soc. españ. fis. quim. 32, 265 (1934).
} 
Landé $g$-values by reference to the extensive tables given by Kiess and Meggers. ${ }^{10}$

The designation of the term ${ }^{4} \mathrm{~F}_{11 / 2}$ in table 1 is to be regarded as provisional. The $g$-values given for higher even levels are only preliminary values and probably have an average error of at least 0.1 ; but, on the basis of the data, they are fairly consistent. The identification of the level $08_{01 / 2}$ with ${ }^{4} \mathrm{P}_{01 / 2}$, and the level $13_{01 / 2}$ with ${ }^{4} \mathrm{D}_{01 / 2}$ is conjectural, but if correct, all three quartet terms, ${ }^{4} \mathrm{P},{ }^{4} \mathrm{D}$, and

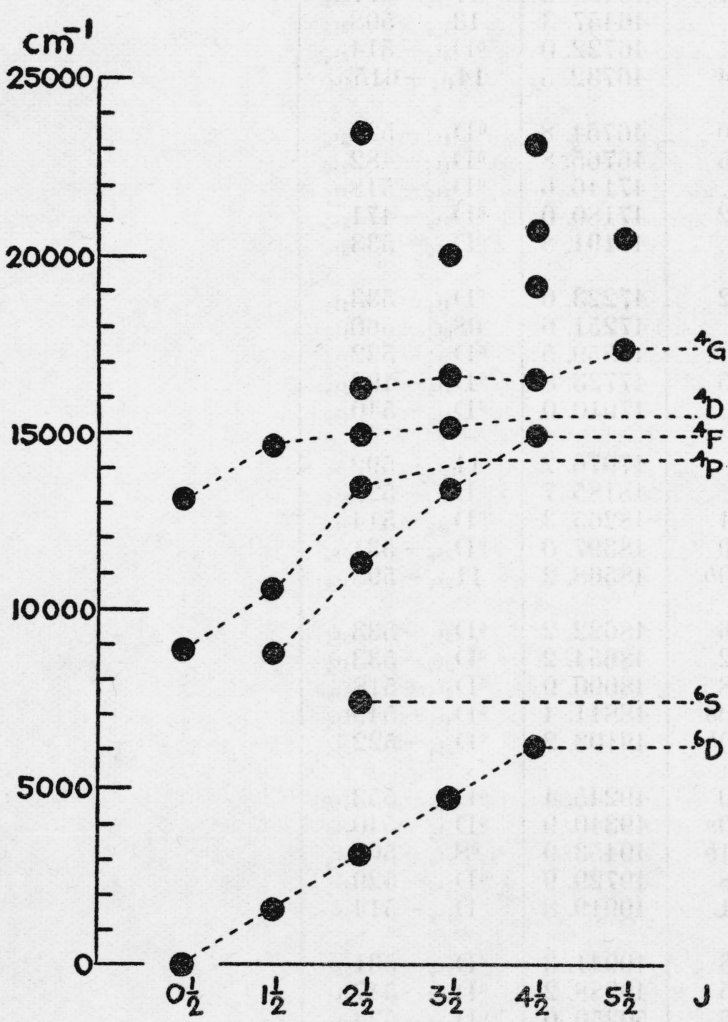

Figure 1.-Low and metastable terms of $\mathrm{W}$ II with probable multiplet arrangement. ${ }^{4} \mathrm{~F}$, can be filled out from the neighboring terms in a very simple manner and with regularintervals. All the levels of ${ }^{4} \mathrm{G}$ are next in line, ending with $17_{51 / 2}$. These conjectures are illustrated in figure 1. The four quartets would account for all the unidentified levels below $19_{41 / 2}$.

According to the rule of Meggers and Scribner, ${ }^{11}$ the raie ultime of W II should be represented by the term combination $5 d^{4} 6 s \quad{ }^{6} \mathrm{D}_{41 / 2}-5 d^{4} 6 p$ ${ }^{6} \mathrm{~F}_{51 / 2}^{\circ}$. The theoretical Zeeman effect for this transition in LScoupling is $(0.05$, $0.15, \ldots ..) \mathbf{1 . 0 0}$, $1.10, \ldots$ The line at $2204.49 \mathrm{~A}$ has the observed Zeeman pattern (0.00) 0.96, and it has outstanding intensity in both arc and spark sources.

Since it also represents the transition ${ }^{6} \mathrm{D}_{41 / 3}-514_{51 / 2}$, this line is in all probability the true raie ultime of $\mathrm{W}$ ir.

\section{NEW LEVELS AND g-VALUES FOR THE ARC SPECTRUM W I}

After the discovery of the low terms of W I, Laporte has continued the analysis of this spectrum with the collaboration of J. E. Mack. I have had the opportunity of being associated with Professor Mack in this work for several months, at the University of Wisconsin, and have had access to all their results. When the new wave-length measurements described above had been completed, some of the levels that had appeared to be doubtful on the basis of the older data,

10 C. C. Kiess and W. F. Meggers, BS J. Research 1, 641 (1928) RP23.

11 W. F. Meggers and B. F. Scribner, NBS J. Research 13, 657 (1934) RP732. 
were verified, and others were rejected. This brought the number of odd levels known in 1934 to 170.

TABLE 4.-New levels for the arc spectrum $\mathrm{W}_{\mathrm{I}}$

\begin{tabular}{|c|c|c|c|c|c|}
\hline$J$ & Term value & Remarks & $J$ & Term value & Remarks \\
\hline $\begin{array}{l}2 \ldots \\
0 \ldots\end{array}$ & $\begin{array}{l}20,983.08 \\
41,965.14 \\
49,529.55 \\
45,374.02 \\
48,389.95 \\
49,443.66 \\
53,042.02 \\
54,941.06 \\
55,859.36 \\
\\
21,448.70 \\
33,943.98 \\
35,311.46 \\
49,151.92 \\
51,182.36 \\
51,693.90 \\
52,064.15 \\
52,152.62 \\
53,959.38 \\
54,859.22 \\
55,032.72 \\
55,084.05 \\
55,619.70 \\
55,835.20 \\
59,422.00 \\
59,999.10 \\
42,514.09 \\
49,270.17 \\
51,072.24 \\
52,015.30 \\
52,255.78 \\
52,943.50 \\
53,345.60 \\
53,390.48 \\
54,556.50 \\
55,389.32 \\
55,546.08 \\
56,108.51 \\
56,484.30 \\
56,717.14 \\
58,091.56 \\
58,206.00 \\
58,644.00\end{array}$ & $\begin{array}{l}\text { LM give } J=4 \\
\text { LM give } J=4\end{array}$ & 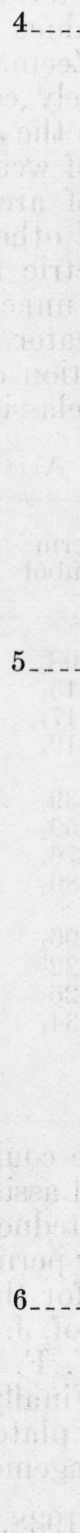 & $\begin{array}{l}38,001.10 \\
40,233.93 \\
40,583.01 \\
43,720.86 \\
47,689.29 \\
49,148.00 \\
50,284.72 \\
52,059.74 \\
52,992.70 \\
53,118.28 \\
54,118.78 \\
54,911.63 \\
55,043.36 \\
55,867.28 \\
55,955.36 \\
56,174.67 \\
56,255.70 \\
56,831.64 \\
57,803.66 \\
58,777.78 \\
59,171.70 \\
60,385.01 \\
26,676.38 \\
45,789.08 \\
46,506.32 \\
50,806.06 \\
51,290.73 \\
52,395.46 \\
52,774.10 \\
53,194.25 \\
54,310.30 \\
55,009.20 \\
55,455.28 \\
55,492.18 \\
55,795.60 \\
55,987.86 \\
56,280.46 \\
57,143.47 \\
57,560.80 \\
58,179.37 \\
58,562.62 \\
58,903.94 \\
59,263.60 \\
59,673.30 \\
38,203.04 \\
52,855.95 \\
53,228.38 \\
54,733.32 \\
56,526.58 \\
57,919.12 \\
59,128.76 \\
59,410.48 \\
5\end{array}$ & $\begin{array}{l}(?) \\
(5 ?) \text { give } J=3 \text { or } 4 \\
\text { LM give }\end{array}$ \\
\hline & & & & $43,411.40$ & (?) \\
\hline
\end{tabular}


Further analysis of the new data yielded the 88 new odd levels given in table 4, as well as one even level. Later it was learned that a number of the new levels were independently discovered by Poggio ${ }^{12}$ with some differences of $J$-value. The question-marked levels require additional observations in other regions of the spectrum in order to be definitely established.

New $g$-values for 37 odd levels, established by Laporte and Mack, are given in table 5 . The terms are designated by the first three figures of their numerical values with subscripts denoting their inner quantum numbers. The Zeeman patterns measured in the new region were almost exclusively combinations with the low D and S terms. A few corrections to the $J$-values in table 4 may be necessary when more Zeeman effects of weaker lines become available.

A considerable number of arc lines remain unclassified; some of which are quite intense and others of which show absorption in the under-water spark ${ }^{13}$ or electric furnace. ${ }^{14}$ The origin of the dozen absorption lines that remain unaccounted for perhaps lies in relatively high levels with $J$-value greater than the ${ }^{7} \mathrm{D}_{4}$, as was indicated in a previous note. ${ }^{15}$ Reobservation of the region from 3100 to $5500 \mathrm{~A}$ will help to give a basis for classifying these lines.

TABLE 5.-New g-values for arc levels

\begin{tabular}{|c|l||l|l||l|l|}
\hline $\begin{array}{c}\text { Term } \\
\text { symbol }\end{array}$ & $g$-value & $\begin{array}{c}\text { Term } \\
\text { symbol }\end{array}$ & $g$-value & $\begin{array}{c}\text { Term } \\
\text { symbol }\end{array}$ & $g$-value \\
\hline & 1.63 & $408_{2}$ & 1.2 & $438_{3}$ & 1.21 \\
$361_{1}$ & 0.8 & $415_{2}$ & 1.02 & $440_{3}$ & 1.14 \\
$377_{1}$ & 0.90 & $417_{2}$ & 1.20 & $463_{3}$ & 1.3 \\
$391_{1}$ & 1.4 & $419_{2}$ & 0.96 & $382_{4}$ & 1.28 \\
$396_{1}$ & 1.5 & $439_{2}$ & 1.33 & $387_{4}$ & 1.07 \\
$404_{1}$ & 1.25 & $450_{2}$ & 1.20 & $397_{4}$ & 1.18 \\
$407_{1}$ & 1.72 & $376_{3}$ & 1.10 & $402_{4}$ & 1.32 \\
$422_{1}$ & 1.1 & $380_{3}$ & 1.1 & $405_{4}$ & 1.26 \\
$425_{1}$ & 1.48 & $396_{3}$ & 1.56 & $411_{4}$ & 1.30 \\
$438_{1}$ & 1.6 & $422_{3}$ & 1.36 & $452_{4}$ & 1.08 \\
$366_{2}$ & 1.24 & $426_{3}$ & 1.18 & $466_{4}$ & 1.3 \\
$374_{2}$ & 1.31 & $434_{3}$ & 1.24 & $469_{4}$ & 1.3 \\
$390_{2}$ & & & & $465^{5}$ & 1.4 \\
& & & & & \\
\hline
\end{tabular}

The work described above could not have advanced to its present state without the advice and assistance of others. It is a pleasure to acknowledge here my indebtedness to Rev. Joseph F. Carroll, S. J., of Marquette University, for permission to work in his laboratory and to express my appreciation for the many courtesies he has extended to me. The assistance of Prof. J. E. Mack with the Zeeman and other calculations, and that of W. F. Meggers with the term analysis, is gratefully acknowledged. Finally, to C. C. Kiess special thanks are due for making most of the plates used in this investigation and for his constant aid and encouragement throughout.

Washington, March 10, 1938.

12 F. Poggio, Anales soc. españ. fis. quim. 33, 171 (1935)

13 W. F. Meggers, Unpublished National Bureau of Standards observations.

14 A. S. King, Astrophys. J. 75, 379 (1932).

${ }_{15}$ D. D. Laun, Phys. Rev. 48, 572 (1935). 